\title{
More Than Brides Alliance (MTBA): Mariage n'est pas un jeu d’enfat, Rapport de référence Mali
}

Andrea J. Melnikas

Population Council

Sajeda Amin

Population Council

Sarah Engebretsen

Mouhamadou Gueye

Follow this and additional works at: https://knowledgecommons.popcouncil.org/departments_sbsr-pgy

Part of the Demography, Population, and Ecology Commons, Family, Life Course, and Society Commons, International Public Health Commons, and the Maternal and Child Health Commons How does access to this work benefit you? Let us know!

\section{Recommended Citation}

Melnikas, Andrea J., Sajeda Amin, Sarah Engebretsen, and Mouhamadou Gueye. 2017. "More Than Brides Alliance (MTBA): Mariage n'est pas un jeu d'enfat, Rapport de référence Mali," Baseline report. New York: Population Council. 


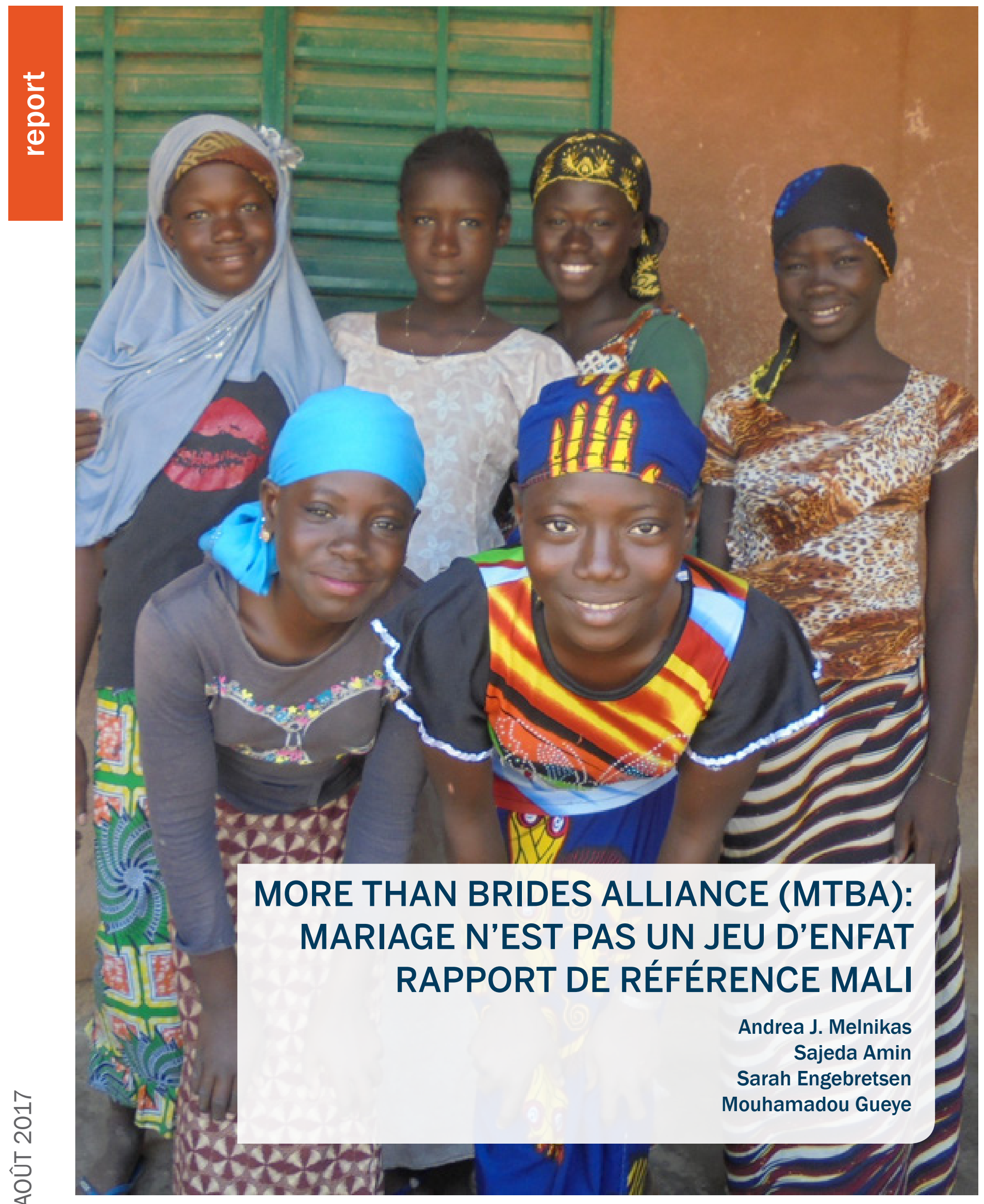

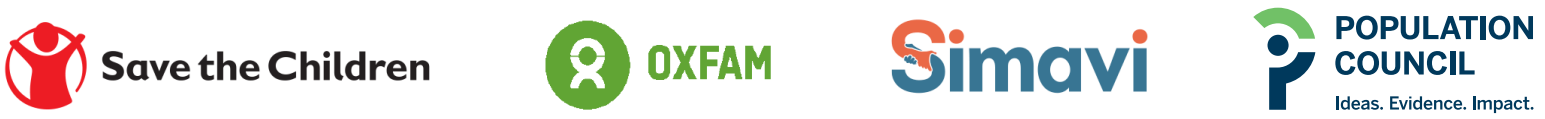




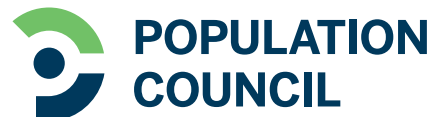 \\ Ideas. Evidence. Impact.}

Population Council s'attelle aux questions cruciales de la santé et du développement - qu'il s'agisse d'endiguer la progression du VIH, d'améliorer la santé reproductive et d'offrir aux jeunes la promesse d'une vie satisfaisante et productive. Par ses travaux de recherche en biomédecine, en sciences sociales et en santé publique dans 50 pays, Population Council s'efforce d'apporter avec ses partenaires des solutions qui conduisent à des politiques, des programmes et des technologies plus efficaces, pour améliorer la vie dans le monde. Fondé en 1952, Population Council est une organisation non gouvernementale à but non lucratif qui siège à New York sous la conduite d'un conseil d'administration international.

\section{Population Council}

1 Dag Hammarskjold Plaza

New York, NY 10017

États-Unis

Tél : +12123390500

Fax : +12127556052

Courriel : pubinfo@popcouncil.org

popcouncil.org

Reference suggérée : Melnikas Andrea J., Sajeda Amin, Sarah Engebretsen et Mouhamadou Gueye. 2017. More Than Brides Alliance (MTBA): Mariage n'est pas un jeu d'enfat Rapport de référence Mali. New York: Population Council.

Crédit photo: Save the Children International Mali

Les rapports de référence de l'Alliance "Plus qu'une épouse " (More Than Brides Alliance) sont disponibles pour l'Inde, le Malawi, le Mali, et le Niger.

(C) 2017 The Population Council, Inc.

Translated to French from “More Than Brides Alliance: Baseline Report, Mali,” 2017, New York: Population Council. 


\section{Table des matières}

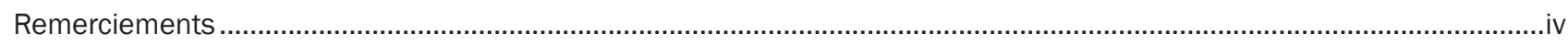

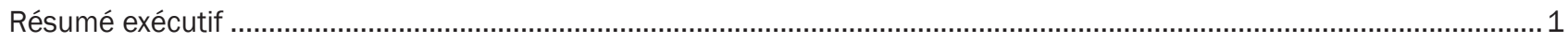

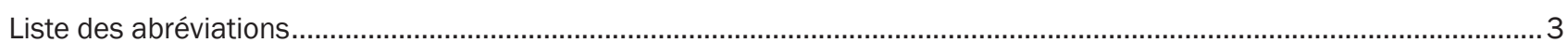

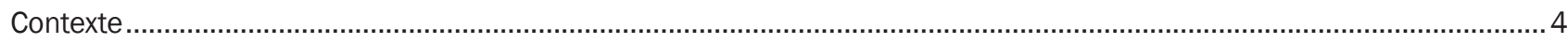

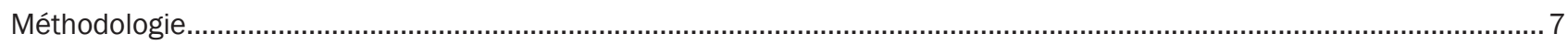

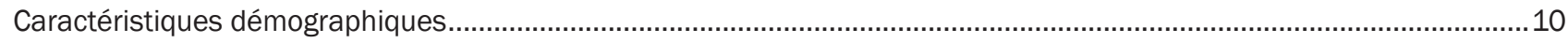

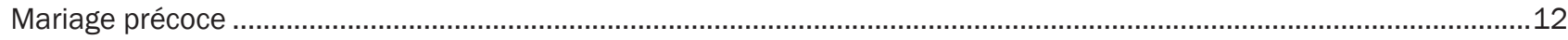

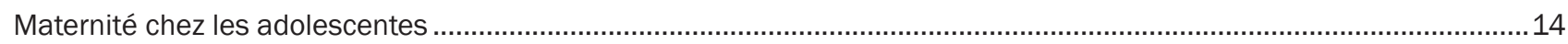

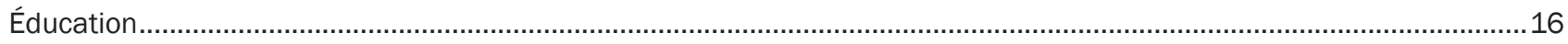

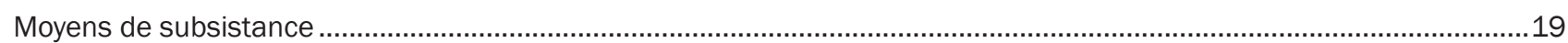

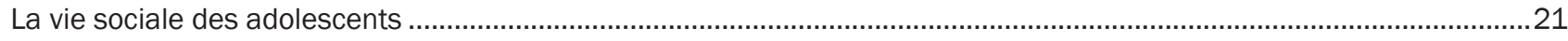

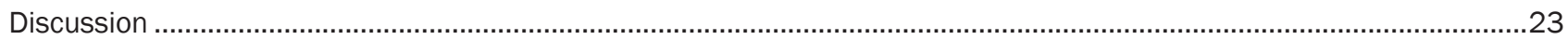

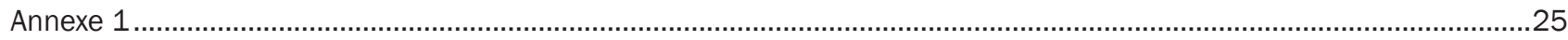

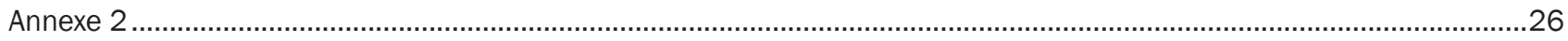

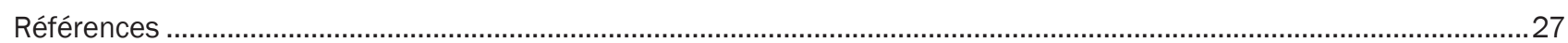




\section{Remerciements}

Plusieurs personnes ont contribué à cette étude de base du Mali. Nous leur sommes reconnaissants pour leurs efforts.

Nous tenons à remercier les membres de l'Alliance "Plus qu'une épouse " "More than Brides Alliance", MTBA), y compris "Save the Children International/Pays-Bas", Oxfam Novib et Simavi. Au Mali, Save the Children International/Pays-Bas et Oxfam Novib mettent actuellement en œuvre des programmes visant à retarder l'âge du mariage et à améliorer la situation des adolescentes, en collaboration avec les partenaires locaux -- AGIR, SORO, Fawe Mali et Wale. Nous attendons les résultats de ces interventions avec intérêt.

Nous remercions également le Ministère néerlandais des Affaires étrangères pour le soutien qu'il apporte à l'Alliance " Plus qu'une épouse ", MTBA plus particulièrement dans les volets recherche et évaluation du projet.

Nous souhaitons remercier nos collègues de LASDEL qui ont supervisé la collecte des données sur le terrain, y compris Mouhamadou Gueye, Bourame Fane et Sekou Fofana. Nous avons eu la chance de travailler avec une équipe talentueuse qui a joué un rôle fondamental dans la conduite du recensement des ménages et de l'étude de référence avec les adolescentes et les jeunes femmes.

Enfin, nous exprimons notre reconnaissance aux participants à l'étude, particulièrement aux adolescentes et aux jeunes femmes qui ont partagé leurs expériences, leurs pensées et leurs ambitions avec l'équipe de recherche. Nous les remercions pour leur participation, sans laquelle ce rapport n'aurait pas pu voir le jour. 


\section{Résumé exécutif}

Dans le cadre d'une évaluation des interventions visant à retarder l'âge du mariage, nous avons mené des enquêtes de référence sur les adolescentes dans les régions de Sikasso et Ségou au Mali. Les objectifs de l'étude de référence étaient les suivants : 1) fournir un point de référence permettant de mesurer les changements découlant de l'intervention à mi-parcours et à la fin de l'étude ; 2) fournir des renseignements sur la situation et le contexte actuels des adolescentes dans des zones précises au Mali afin de documenter l'intervention visant à retarder le mariage ; et 3 ) identifier les thèmes nécessitant un examen approfondi à l'aide de la recherche qualitative. L'étude de référence s'est déroulée en partenariat avec un groupe de recherche local basé à Bamako : le Centre d'étude et de recherche sur l'information en population et en santé (CERIPS). La collecte des données de référence comprenait un recensement des ménages $(n=2831)$ qui a recueilli des données sur les filles et les femmes âgées de 10 à 21 ans qui vivaient au sein du ménage, et des études de référence sur des adolescentes et des jeunes femmes âgées de 12 à 19 ans ( $n=855$ qui ont recueilli des données sur le statut matrimonial et les antécédents, le niveau d'éducation, les connaissances et expériences liées à la santé sexuelle et reproductive, les résultats d'apprentissage, l'historique de la migration, les moyens de subsistance et les relations sociales. Les études ont été réalisées dans des villages d'intervention $(n=20)$ et dans des villages de comparaison $(n=20)$, qui ont été sélectionnés sur la base de critères identiques.

Notre échantillon comprenait 300 filles dans la région de Sikasso et 555 filles dans la région de Ségou, dont l'âge moyen était de 15,1 ans à Sikasso et de 15,3 à Ségou. La proportion des filles qui étaient ou avaient été mariées était un peu plus élevée à Sikasso (15,4\%) qu'à Ségou (13,9\%). Parmi les participantes âgées de 15 à 19 ans, 25,2\% ont signalé qu'elles étaient ou avaient été mariées, avec une proportion plus élevée à Sikasso (27,9\%) qu'à Ségou $(23,8 \%)$.

Dans l'ensemble, 59,6\% des participantes n'étaient pas scolarisées. Cette proportion était plus élevée à Ségou $(64,1 \%)$ qu'à Sikasso (51,3\%). Parmi les participantes, $62,7 \%$ ont déclaré avoir été scolarisées, avec 6,3 années de scolarité en moyenne. Nous avons effectué une courte évaluation de leur niveau d'alphabétisation et nos résultats montrent que malgré le fait que près des deux tiers des filles étaient allées à l'école à un moment donné, seulement 19,0\% des filles à Sikasso et 16,3\% à Ségou ont réussi à lire deux phrases complètes en français.

Nous avons étudié les opportunités de subsistance pour comprendre les alternatives aux mariage précoce. Nos résultats indiquent que globalement, près de la moitié des participantes âgées de 12 à 19 ans (48,6\%) ont déclaré avoir déjà travaillé pour gagner de l'argent. Parmi les participantes plus âgées (15 à 19 ans), cette proportion était de $57,0 \%$. Environ un cinquième des participantes $(20,6 \%)$ travaillaient pour gagner de l'argent au moment de l'étude. Pour mieux comprendre la situation des filles dans ces communautés, nous avons également examiné la vie sociale des adolescentes et leurs attitudes sur l'égalité des sexes. Dans les deux régions, les normes sociales liées aux rôles des femmes étaient inquiétantes : 98,8\% des filles ont répondu qu'elles étaient " tout à fait d'accord " ou " d'accord " avec l'idée qu'une femme devait toujours obéir à son mari et 93,9\% étaient " tout à fait d'accord " ou "d'accord " pour qu'une femme supporte la violence afin de préserver l'unité de la cellule familiale.

L'Alliance "Plus qu'une épouse " vise à améliorer la vie des adolescentes et propose des interventions dans plusieurs domaines (la santé reproductive, l'éducation, les normes de genre et les moyens de subsistance, entre autres). Nos résultats suggèrent que des interventions sont nécessaires pour répondre aux vulnérabilités rencontrées par les filles qui vivent dans ces communautés. Par exemple, nos résultats nos résultats indiquent un faible taux de scolarisation, un fort taux d'analphabétisme parmi les jeunes filles ayant participé à l'étude et suggèrent que les pratiques culturelles qui ont une influence sur le mariage précoce, telle que la polygamie, sont répandues. Nos résultats indiquent également que la grossesse précoce est un problème significatif qui s'ajoute à l'accès limité aux services de santé sexuelle et reproductive et aux faibles connaissances dans ce domaine. Toutes ces questions doivent être traitées de manière spécifique par les programmes consacrés aux adolescentes. 
En revanche, de nombreuses filles ont affirmé avoir accès à des opportunités pour subvenir à leurs besoins, représentant une trajectoire alternative et pouvant contribuer à la baisse générale du taux de mariage précoce noté dans les derniers rapports de l'EDS. La collecte de données supplémentaires contribuera à examiner certains de ces thèmes plus en détail et à fournir une compréhension plus nuancée de la situation des adolescentes dans ces communautés. Les résultats de l'étude de référence seront utilisés pour évaluer les changements qui seraient attribuables aux interventions de l'Alliance et pour informer les équipes de programme qui cherchent à mieux comprendre les populations qu'elles servent. 


\section{Liste des abréviations}

$\begin{array}{ll}\text { CERIPS } & \text { Centre d'étude et de recherche sur l'information en population et en santé } \\ \text { CSE } & \text { "Comprehensive Sexuality Education " (Éducation sexuelle complète) } \\ \text { EDSS } & \text { Enquête démographique et de santé } \\ \text { INSTAT } & \text { Institut national des statistiques } \\ \text { LSE } & \text { "Life Skills Education " (Formation en compétences de vie) } \\ \text { MTBA } & \text { "More than Brides Alliance "(Alliance Plus qu'une épouse) } \\ \text { SDSR } & \text { Santé et droits sexuels et reproductifs }\end{array}$




\section{Contexte}

Avec plus de 59\% des femmes âgées de 20 à 24 ans mariées avant l'âge de 18 ans, la prévalence du mariage précoce au Mali est la sixième plus élevée au monde et la troisième plus élevée en Afrique de l'Ouest, après le Niger et la Guinée (EDS, 2013). Le mariage des enfants (défini comme un mariage avant l'âge de 18 ans) a des conséquences négatives sur les filles et les femmes: les filles qui se marient tôt ont souvent des niveaux d'éducation faibles et des compétences limitées (UNICEF 2011). Les écarts d'âge entre les filles qui se marient tôt et leurs époux sont importants, ce qui peut compromettre leur capacité à prendre des décisions liées à la planification familiale et à la maternité (Mensch, Bruce et Greene, 1998). L'indice indice de fécondité au Mali $(6,1)$ compte parmi les plus élevés au monde (EDS, 2013), ce qui suggère que les stratégies de lutte contre la croissance démographique doivent préconiser une stratégie visant à retarder l'âge du premier mariage.

La Diagramme 1 présente quelques différences régionales : Sikasso compte la proportion la plus élevée des femmes âgées de 20 à 24 ans mariées avant l'âge de 15 ans $(27,1 \%)$, tandis que Kayes compte la proportion la plus élevée des femmes âgées de 20 à 24 ans mariées avant l'âge de 18 ans (70,9\%). L'âge moyen du mariage parmi les maliennes (âgées de 20 à 14 ans) est 16,1 ans ; cette

L'Alliance "Plus qu'une épouse " met actuellement en œuvre et évalue l'efficacité d'une série d'interventions visant à retarder l'âge du mariage, notamment sur l'éducation, les opportunités économiques, la promotion des enfants, les services de santé et droits sexuels et reproductifs (SDSR), l'engagement des communautés, ainsi que d'autres sujets. Population Council mène actuellement une étude appariée quasi expérimentale au Mali et au Niger, et un essai randomisé en grappes en Inde et au Malawi afin d'évaluer si ces interventions sur le mariage des enfants améliorent les capacités des jeunes femmes à déterminer le moment de leur mariage et leurs droits à la santé sexuelle et reproductive dans un environnement favorable, et la mesure dans laquelle elles le font.

DIAGRAMME 1. Pourcentage de filles mariées avant l'âge de 15 et 18 ans chez les femmes âgées de 20 À 24 ans (EDS 2012/13)

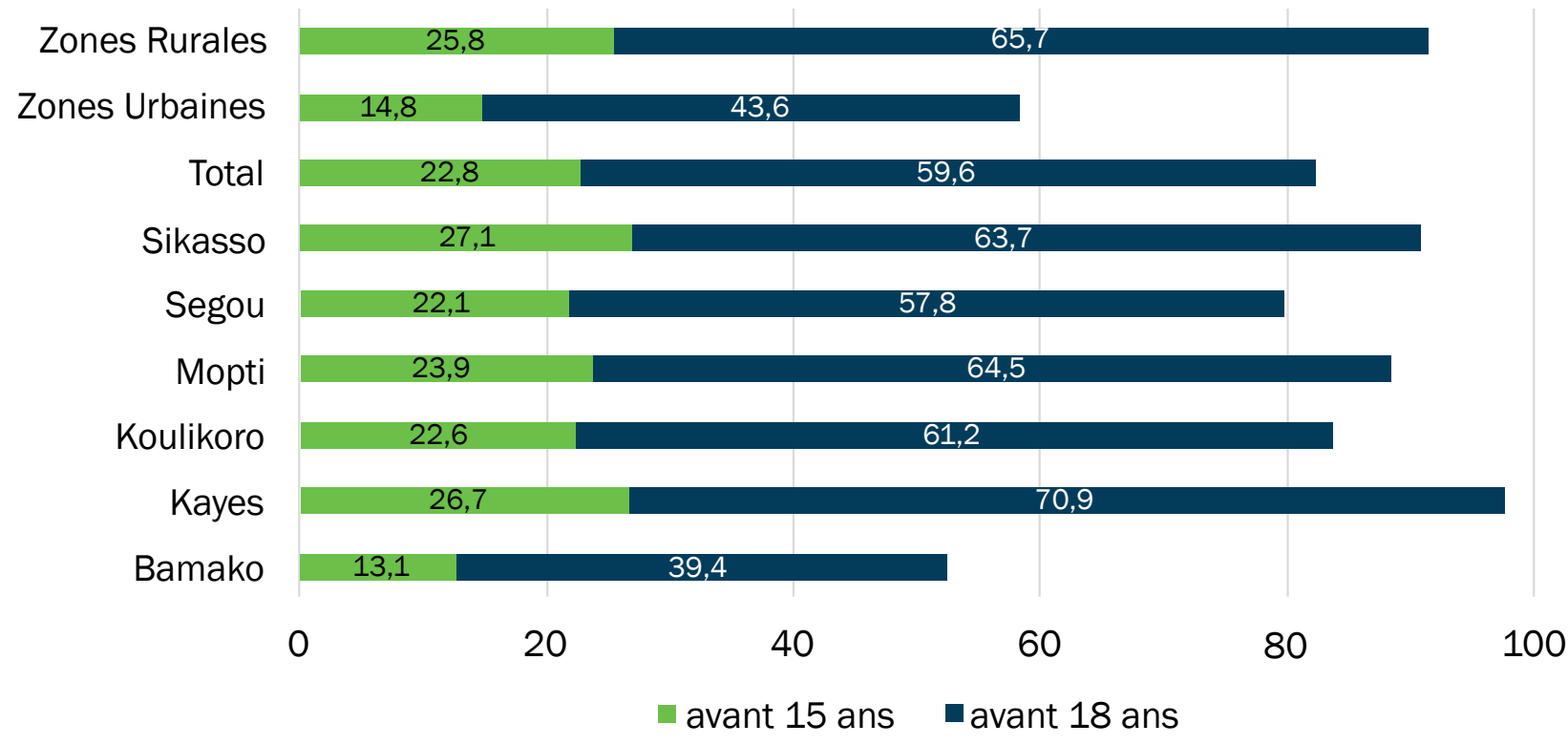


TABLEAU 1. Âge moyen (médian) au premier mariage, femmes âgées de 20 à 24 ans (EDS, 2012/13)

\begin{tabular}{|c|c|}
\hline Région & Moyenne (Médian) \\
\hline Bamako & $16,7(17)$ \\
\hline Kayes & $15,5(16)$ \\
\hline Koulikoro & $16,2(16)$ \\
\hline Mopti & $16,0(16)$ \\
\hline Ségou & $16,3(16)$ \\
\hline Sikasso & $15,9(16)$ \\
\hline Total & $16,1(16)$ \\
\hline
\end{tabular}

moyenne étant inférieure à 16 ans à Kayes et Sikasso. À Bamako, la région la plus urbaine du pays, l'âge moyen au premier mariage est le plus élevé (16,7 ans) (Tableau 1).

Le taux des mariages d'enfants est le plus bas dans la région la plus urbanisée qui entoure Bamako. II existe des différences significatives sur l'âge du mariage entre les zones urbaines et rurales au Mali, les jeunes femmes vivant des les zones rurales étant plus susceptibles de se marier tôt. Plus d'un quart des femmes âgées de 20 à 24 ans vivant dans des zones rurales ont déclaré qu'elles s'étaient mariées avant l'âge de 15 ans (25,8\%) par rapport à 14,8\% dans les zones urbaines. Cet écart double en tenant compte des femmes mariées avant l'âge de 18 ans : 65,7\% des femmes âgées de 20 à 24 ans dans les zones rurales se marient avant l'âge de 18 ans, par rapport à 43,6\% dans les zones urbaines.

La polygamie et la dot sont des pratiques maritales très répandues au Mali et peuvent avoir des conséquences importantes sur le mariage précoce. Selon les données de l'EDS, 35\% de toutes les femmes mariées déclarent être dans une union polygame (EDS 2012/13). Certaines données suggèrent que les femmes qui se marient tôt sont plus susceptibles de se retrouver dans une union polygame, sachant qu'elles jouent le rôle de femme " supplémentaire ". Un rapport du Centre international de recherche sur les femmes (ICRW) " (2015) a révélé que 36,2\% des maliennes mariées avant l'âge de 18 ans étaient dans des unions polygames par rapport à $25,7 \%$ des femmes qui se sont mariées après l'âge de 18 ans. Certaines variations des taux de prévalence de la polygamie sont observées entre les zones urbaines et rurales et entre les différentes régions du pays : la polygamie est plus fréquente dans les zones rurales (38\% des femmes déclarent être en union polygame) que dans les zones urbaines (22\%). La pratique est un
DIAGRAMME 2. Âge médian au premier mariage (toutes les femmes) par niveau de scolarité (EDS 12/13)

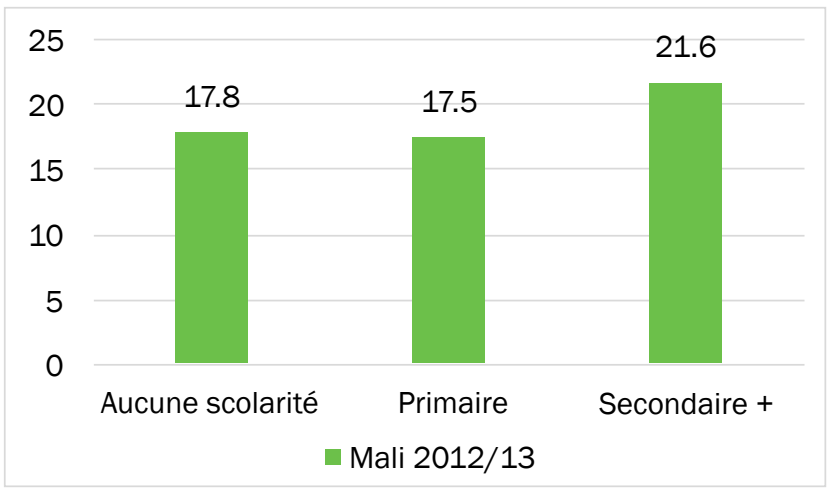

fait plus courant dans les régions de Sikasso (44\%), Mopti (38\%) et Kayes (37\%), où l'âge moyen au premier mariage est le plus faible (EDS 2013).

Au Mali, le taux de scolarisation des adolescents, calculé selon le taux d'achèvement des études primaires, Diagramme parmi les plus bas au monde (UNESCO, 2014) et le décrochage scolaire se produit très tôt pour les enfants des deux sexes. Parmi les enfants âgés de 10 à 14 ans, le pourcentage des filles non scolarisées est de 57,8\% au niveau national, et encore plus élevé dans les zones rurales $(66,1 \%)$. Parmi les garçons, ces chiffres sont également élevés au niveau national : 49,1\% des enfants âgés de 10 à 14 ne sont pas scolarisés. Parmi les adolescentes âgées de 15 à 19 ans, les chiffres sont bien pires : les trois-quarts $(75,4 \%)$ des filles âgées de 15 à 19 ans au niveau national et $84,8 \%$ de ces filles dans les zones rurales ne sont pas scolarisées. Dans certaines régions, plus de $90 \%$ des filles âgées de 15 à 19 ans ont déjà quitté l'école (EDS 2006, données non publiées).

Le lien entre la scolarisation des filles et le retard de l'âge du mariage est bien documenté au Mali, et la scolarisation des filles est largement reconnue comme un facteur de protection contre le mariage précoce. Comme le montre la Diagramme 2, l'âge médian du mariage augmente avec le niveau de scolarité. Parmi les femmes ayant déclaré un niveau de scolarité dans le secondaire ou plus, le taux médian de l'âge au premier mariage médian était de 21 , 6 ans, par rapport à 17, 5 ans parmi celles qui avaient atteint un niveau primaire, et 17,8 ans parmi les filles sans aucune scolarité formelle. 


\section{Intervention}

L'Alliance "Plus qu'une épouse " (More Than Brides Alliance - MTBA) est un programme mis en œuvre par Save the Children International/Pays-Bas, Oxfam Novib et Simavi, qui vise à améliorer la situation des adolescentes dans cinq pays : I'Inde, le Malawi, le Mali, le Niger et le Pakistan.

Globalement, les programmes MTBA s'articulent autour de cinq domaines de résultats clés :

- Autonomiser les adolescentes exposées au risque de mariage précoce et les adolescents déjà mariées, en leur offrant une formation en compétences de vie, une éducation sexuelle complète et des informations sur la santé et les droits sexuels et reproductifs (SDSR);

- Offrir des alternatives au mariage précoce et réduire l'impact sur les filles mariées en améliorant l'accès des filles et de leur famille à l'éducation, aux opportunités économiques et aux systèmes de protection de l'enfance ;

- Accroître l'accès des jeunes aux services de SDSR ;

- Changer les normes sociales ;

- $\quad$ Influencer les cadres juridiques et politiques.

Au Mali, le programme MTBA est actuellement mis en œuvre dans les régions de Sikasso et Ségou par Save the Children et Oxfam Novib, respectivement, en coopération avec des ONG locales. En tant que partenaire de la recherche et du programme d'apprentissage MTBA, le Population Council mène actuellement une évaluation des interventions en Inde, au Pakistan, au Malawi, au Mali et au Niger.

\section{Objectifs de l'étude}

Les objectifs de cette étude de référence visaient à : 1) fournir un point de référence permettant de mesurer les changements découlant de l'intervention à mi-parcours et à la fin de l'étude ; 2) fournir des renseignements sur la situation et le contexte actuels des adolescentes dans des zones sélectionnées au Mali afin de documenter l'intervention ; et 3) identifier les thèmes nécessitant un examen approfondi à l'aide de la recherche qualitative. 


\section{Méthodologie}

L'étude de référence comprenait les éléments de collecte de données suivants :

- Le recensement des ménages dans une sélection de villages d'intervention et de comparaison, accompagné de la collecte des indicateurs clés de résultats pour les filles et les femmes âgées de 10 à 21 ans, y compris leur statut matrimonial, scolaire, maternel et professionnel

- L'étude de référence parmi les filles âgées de 12 à 19 ans dans une sélection des communautés d'intervention et de comparaison. Les questions portaient sur :

- Les caractéristiques générales

- La migration

- La scolarisation et les expériences à l'école, y compris une évaluation des compétences en lecture et en calcul

- Les connaissances sur la santé reproductive

- Le mariage et la dot

- L'expérience sexuelle

- La mobilité

- Le contexte social

- L'égalité des genres

- Les moyens de subsistance

La programme MTBA vise à améliorer la vie des adolescentes, notamment en augmentant le taux de scolarisation et le niveau d'éducation, en améliorant les résultats en matière de santé reproductive et en encourageant les filles à utiliser leur libre arbitre pour prendre des décisions concernant leurs vies, y compris les décisions liées au mariage. Les principaux résultats d'intérêt portent sur les indicateurs tels que la proportion de filles mariées avant l'âge de 18 ans, la proportion de filles enceintes avant l'âge de 18 ans, ou encore la proportion de filles scolarisées. Plusieurs points abordés dans l'étude sont directement liés à ces résultats (par exemple, les questions relatives à l'âge au premier mariage), tandis que d'autres sont moins directement liés mais peuvent avoir une influence (par exemple, la migration peut rendre les filles plus ou moins vulnérables à des effets néfastes en fonction des circonstances entourant leur migration). Nous avons recueilli des données sur les indicateurs qui nous permettront de mesurer l'impact du programme et de mieux comprendre le contexte et les contraintes entourant les résultats clés afin de documenter la conception et la mise en œuvre du programme.

L'étude a été mise en œuvre dans les régions de Ségou et Sikasso au Mali par le Centre d'étude et de recherche sur l'information en population et en santé (CERIPS) basé à Bamako. La Diagramme 3 montre les zones de collecte des données de référence de l'étude.

Revue éthique : Nous avons obtenu l'accord du Comité d'examen institutionnel (Institutional Review Board) de Population Council, et du Comité d'éthique de l'INRSP et du Ministère de la Santé et de l'Hygiène publique du Mali à Bamako pour mener cette recherche.

Taille de l'échantillon : Nous avons effectué des estimations de la taille de l'échantillon à l'aide du logiciel Optimal Design avec pour hypothèse de départ la moitié des zones de collecte de données seraient désignées comme zones de comparaison. L'Annexe 1 présente nos calculs et nos hypothèses. Nous avons estimé qu'il sera possible d'atteindre une puissance statistique de $80 \%$ avec 40 grappes (zones de recensement) et 20 filles par grappe pour un échantillon total de 800 filles âgées de 12 à 19 ans.

Conception de l'essai : Nous avons utilisé un modèle quasi-expérimental ${ }^{1}$ en sélectionnant des zones d'intervention et de comparaison des indicateurs clés selon les recommandations des partenaires chargés de la mise en $œ u v r e$ du programme. En raison des plans de mise en œuvre du programme, la réalisation d'une conception randomisée en grappes n'a pas été possible.

1 Dans les études quasi-expérimentales, le traitement des données n'est pas attribué de manière aléatoire. En revanche, dans les modèles expérimentaux, le traitement est aléatoire et l'on suppose que les groupes d'intervention et de comparaison sont similaires au début de l'intervention. 


\section{Collecte des données}

Sélection des sites

Les zones d'intervention: Les partenaires d'exécution du programme MTBA (Save the Children et Oxfam Novib) ont sélectionné les villages d'intervention. Les critères de sélection étaient les suivants :

1) L'existence d'une infrastructure scolaire (une école primaire et une école secondaire) ;

2) L'existence d'une infrastructure de santé (un centre de santé communautaire, un dispensaire, une maternité et une pharmacie) ;

3) La taille de la population et le nombre estimé d'adolescentes ; ${ }^{2}$

4) L'accessibilité pendant toutes les saisons de l'année ;

5) La mise en œuvre antérieure d'un programme sur le mariage des enfants qui était un précurseur de l'intervention MTBA ${ }^{3}$ et/ou un partenariat sur la qualité de l'éducation, la protection, et les droits humains dans la région de Ségou

6) L'absence d'autres interventions sur le mariage des enfants

Sur la liste des villages d'intervention sélectionnés par le programme MTBA ( $n=43)$, nous avons sélectionné un échantillon aléatoire de 20 villages constituant les villages d'intervention pour l'étude de référence. II convient de noter que parmi ces 43 villages d'intervention, 31 villages sont desservis par Oxfam Novib et situés dans la région de Ségou, et 12 villages sont situés dans la région de Sikasso, desservie par Save the Children.

Les zones de comparaison : Compte tenu de la spécificité de la sélection des villages d'intervention, notre objectif était de sélectionner les zones de comparaison qui répondaient dans la mesure du possible aux mêmes critères de sélection. Nous avons demandé aux partenaires d'exécution de fournir une liste des villages situés dans les mêmes districts que les villages d'intervention qui répondaient aux critères de sélection. II convient de noter qu'il n'a pas été possible de répondre à tous les critères de sélection. Par exemple nous n'avons pas de villages de comparaison
DIAGRAMME 3. Zones de collecte de données pour l'étude de base

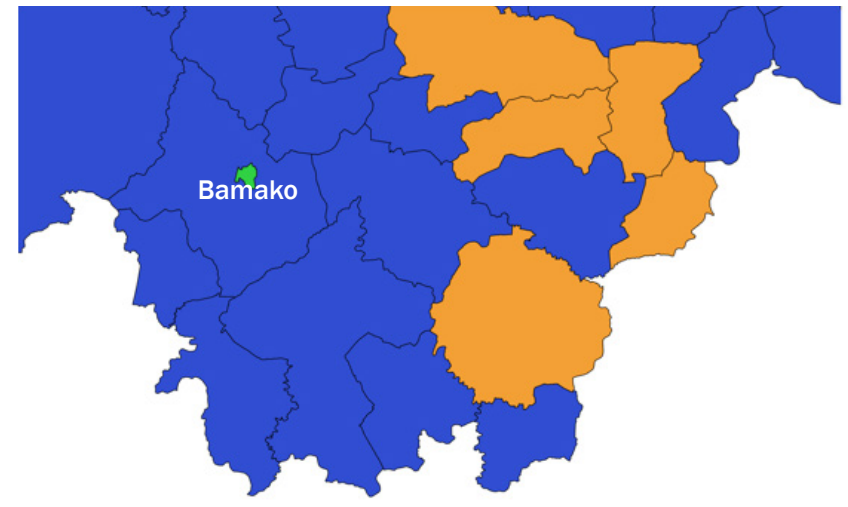

qui avaient déjà mis en œuvre un programme précurseur de l'intervention MTBA. Sur la base de la liste des zones de comparaison adaptées fournie par les partenaires $(n=245)$, nous avons supprimé les villages dont la taille de la population était trop élevée ou trop faible pour permettre une comparaison avec les villages d'intervention $(n=54)$ en fonction de la taille de la population dans les villages d'intervention (entre 1100 et 12000 habitants). Nous avons ensuite sélectionné de manière aléatoire 20 villages parmi les villages restants $(n=191)$ pour servir de zones de comparaison.

\section{Collecte des données}

Les zones de recensement : Bien que la randomisation ait été utilisée au niveau des villages, afin de standardiser la collecte des données, nous avons sélectionné des zones de recensement ${ }^{4}$ représentant les limites pour la collecte des données des listes des ménages. Nous avons obtenu les cartes de ces zones de recensement auprès de l'Institut national des statistiques (INSTAT) à Bamako. Pour les villages sélectionnés comprenant plusieurs zones de recensement, nous avons sélectionné une de ces zones de manière aléatoire pour inclusion dans la collecte des données de référence. Une fois sélectionnée, tous les ménages vivant dans cette zone de recensement ont fait l'objet d'une visite et les données sur les femmes âgées de 10 à 21 ans vivant dans ce ménage ont été recueillies auprès d'un membre adulte du ménage.

\footnotetext{
${ }^{2}$ Les zones d'intervention comptaient une population variant entre 1100 et 12000 personnes.

${ }^{3}$ Le programme de "My Rights My Voice " [Mes Droits, Ma Voix] a été mis en place dans plusieurs pays, y compris au Mali. Pour plus d'informations, veuillez consulter : http://policy-practice.oxfam.org.uk/our-work/youth/youth-participation/my-rights-my-voice

${ }^{4}$ Les zones d'énumération sont des zones géographiques délimitées dans le recensement.
} 
Les participants: Tous les ménages vivant dans la zone de recensement sélectionnée étaient admissibles pour inclusion dans l'étude. Lorsque le ménage ne signalait aucune adolescente âgée de 10 à 21 ans, la liste était relativement courte. À partir de la base de sondage des ménages, nous avons sélectionné au hasard des ménages où vivaient des jeunes femmes âgées de 12 à 19 ans pour participer à l'étude de référence Une seule femme par ménage a été sélectionnée pour inclusion dans l'étude de référence.

\section{Le recensement des ménages et l'étude de référence}

Le recensement des ménages ainsi que la collecte des données de l'étude sur les ménages ont été réalisés dans les communautés sélectionnées dans les régions de Ségou et Sikasso pendant les mois de juillet et août 2016. Des enquêtrices ont été sélectionnées à partir d'une liste d'enquêteurs ayant déjà travaillé avec le CERIPS. Quinze enquêtrices ont été désignées sur la base de leur expérience dans des études sur les adolescentes et/ou des enquêtes traitant de sujets sensibles. Une formation s'est déroulée à Bamako du 11 au 16 juillet 2016. Les enquêtrices chargées de la collecte des données ont participé à une formation de 6 jours qui comprenait des informations de base sur le projet, les objectifs de l'étude, le contenu du recensement des ménages et des outils de l'étude de référence, la saisie de données, et l'éthique de la recherche et la protection des participants. L'essai préalable des outils s'est déroulé pendant la formation. Trois équipes d'étude ont été mises en place. Chaque équipe était composée d'un chef d'équipe et de quatre enquêtrices.

Les enquêtrices formées par le CERIPS ont collecté les données en binôme, en commençant par le recensement des ménages dans les zones de recensement sélectionnées ( $n=40 ; 20$ zones d'intervention et 20 zones de comparaison), puis en sélectionnant un échantillon aléatoire des participantes ( $n=22$ par zone de recensement) pour l'étude de référence sur la base du recensement des ménages. Toutes les données issues du recensement des ménages ont été enregistrées par voie électronique à l'aide de téléphones portables. Les données de l'étude de référence ont été également enregistrées électroniquement. Au total, 2831 ménages ont été recensés et 855 questionnaires de référence ont été complétés.

Le consentement écrit individuel a été obtenu auprès de l'ensemble des participantes préalablement à la conduite des entretiens. L'autorisation des parents, puis celle des participantes, ont été obtenues pour les adolescentes âgées de moins de 18 ans.

Les données relatives aux indicateurs clés (la proportion de femmes mariées, la proportion de mères adolescentes, la proportion des filles scolarisées) ont été recueillies au cours du recensement des ménages ainsi qu'avec les outils de l'étude de référence. Les données issues du recensement des ménages ont été collectées sur les femmes âgées de 10 à 21 ans au sein du ménage auprès du chef de famille ou d'un autre membre adulte du ménage. Par conséquent, les données issues du recensement des ménages sont susceptibles d'être biaisées. Les données de l'étude de référence ont été recueillies directement auprès des femmes interrogées âgées de 12 à 19 ans.

L'analyse des données

Les données ont été saisies directement dans SurveyCTO via une application mobile et téléchargées sur un serveur sécurisé. Les données ont été téléchargées dans STATA 14.1 pour nettoyage et analyse. L'analyse des données s'est principalement concentrée sur les statistiques descriptives pour les variables d'intérêt. Nous présentons des comparaisons géographiques dans chaque section et l'Annexe 2 présente un tableau comparant les zones d'intervention et de comparaison. 


\section{Caractéristiques démographiques}

Le Tableau 2 présente les données démographiques clés de l'échantillon, par région. Nous avons constaté que les régions de Sikasso et Ségou étaient similaires pour la plupart des variables démographiques d'intérêt, mais la proportion des filles mariées était légèrement plus élevée à Sikasso $(15,4 \%)$ qu'à Ségou $(13,9 \%)$. Des différences notables ont été observées entre les deux régions en termes de religion : la proportion des participantes non-musulmanes était de 23,5\% à Sikasso et de seulement de 4,3\% à Ségou.

La Diagramme 4 montre la répartition par âge des participantes dans l'étude de référence. La proportion plus élevée de filles âgées de 12 ans, et la proportion plus faible de filles âgées de 19 ans sont prévisibles dans une population à la croissance rapide où chaque cohorte de naissance est plus grande que la précédente. L'étude révèle une tendance à arrondir les âges à 12, 15 et 18 ans pour les populations dont les âges ne sont pas connus et doivent être estimés. Le Tableau 2 montre que dans l'ensemble, l'âge moyen des participantes était de 15,1 ans à Sikasso et de 15,3 ans à Ségou. Le pourcentage d'adolescentes mariées est légèrement plus élevé à Sikasso qu'à Ségou. Parmi les filles mariées, l'âge de mariage moyen est similaire dans les deux régions. Le Tableau 2 montre d'autres caractéristiques générales qui sont importantes : l'écrasante majorité des adolescentes ont des parents qui n'ont aucune éducation et des mères moins éduquées que les pères. Environ deux tiers des adolescentes possèdent une carte d'identité nationale et ce taux est similaire dans les deux régions. Sikasso enregistre une proportion de non-musulmanes plus élevée (23,5\%) par rapport à Ségou. Dans les deux, la fécondité est élevée, sachant que les adolescentes interrogées ont une moyenne de 5,3 et 5,0 frères et sœurs à Sikasso et Ségou, respectivement. La répartition des revenus est similaire dans les deux régions avec des proportions égales dans le quintile le plus bas. La question demandant aux participantes si elles avaient toujours vécu dans leur village de résidence suggère que le taux de migration est plus élevé à Ségou.

Nous avons considéré l'ethnicité dans les deux régions, sachant que l'ethnicité peut influencer le mariage précoce. En effet, certains groupes ethniques encouragent la pratique du mariage précoce, voire de la polygamie. Dans l'en- semble, les ethnies les plus fréquemment déclarées étaient les Senoufo/Minianka (35,0\%) les Bambara $(33,1 \%)$, les Peulh (7,2\%) et les Bobo (7,3\%). À Sikasso, la majorité des participantes appartenaient à ethnie Senoufo/Minianka (73,3\%). À Ségou, les Bambara représentaient $47,6 \%$ des participantes, suivis par les Senoufo/Minianka (14,1\%) et les Peulh $(9,2 \%)$ (Tableau 3).

DIAGRAMME 4. Nombre de participantes, par âge

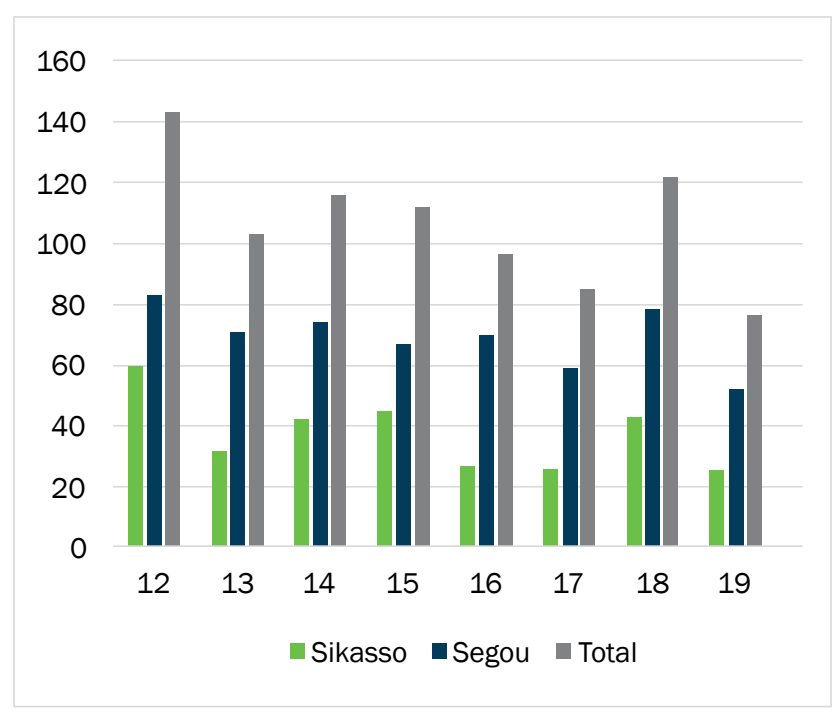


TABLEAU 2. Indicateurs démographiques clés $(n=855)$

\begin{tabular}{|l|c|c|}
\hline & $\begin{array}{c}\text { Sikasso } \\
\mathrm{N}=300\end{array}$ & $\begin{array}{c}\text { Ségou } \\
\mathrm{N}=555\end{array}$ \\
\hline Âge (moyen) & 15,1 & 15,3 \\
\hline $\begin{array}{l}\text { Participantes } \\
\text { actuellement mariées (\%) }\end{array}$ & 15,4 & 13,9 \\
\hline $\begin{array}{l}\text { Participantes } \\
\text { actuellement mariées ou } \\
\text { mariées dans le passé }\end{array}$ & 15,4 & 14,2 \\
\hline $\begin{array}{l}\text { Âge au premier mariage } \\
\text { (moyen) }\end{array}$ & 16,1 & 16,1 \\
\hline Éducation des parents & 91,1 & 85,3 \\
\hline $\begin{array}{l}\text { La mère de la } \\
\text { répondante n'a pas } \\
\text { d'éducation scolaire (\%) }\end{array}$ & 19,0 & 20,5 \\
\hline $\begin{array}{l}\text { Le père de la répondante } \\
\text { n'a pas d'éducation } \\
\text { scolaire (\%) }\end{array}$ & 79,2 & 75,6 \\
\hline $\begin{array}{l}\text { Possède une carte } \\
\text { nationale d'identité }\end{array}$ & 63,0 & 60,3 \\
\hline Est non-musulmane (\%) & 23,5 & 4,3 \\
\hline $\begin{array}{l}\text { Nombre de frères et } \\
\text { sœurs (moyen) }\end{array}$ & 5,3 & 5,0 \\
\hline $\begin{array}{l}\text { Quintile de richesse le } \\
\text { plus bas (\%) }\end{array}$ & $\begin{array}{l}\text { A toujours vécu dans ce } \\
\text { village (\%) }\end{array}$ & \\
\hline
\end{tabular}

TABLEAU 3. Groupe ethnique, par région $(n=852)$

\begin{tabular}{|l|c|c|c|}
\hline & $\begin{array}{c}\text { Sikasso } \\
\mathrm{N}=300\end{array}$ & $\begin{array}{c}\text { Ségou } \\
\mathrm{N}=552\end{array}$ & $\begin{array}{c}\text { Total } \\
\mathrm{N}=852\end{array}$ \\
\hline Bambara & 6,3 & 47,6 & 33,1 \\
\hline Bobo & 6,7 & 7,6 & 7,3 \\
\hline Dogon & 1,3 & 1,3 & 1,3 \\
\hline Malinke & 0,3 & 2,9 & 2,0 \\
\hline Peulh & 3,3 & 9,2 & 7,2 \\
\hline Sarakole/Soninke & 0,3 & 5,8 & 3,9 \\
\hline Senoufo/Minianka & 73,3 & 14,1 & 35,0 \\
\hline Sonrai & 0,0 & 2,5 & 1,6 \\
\hline Tamachek/Bella & 0,0 & 1,1 & 0,7 \\
\hline Other & 8,3 & 7,8 & 8,0 \\
\hline
\end{tabular}

Veuillez noter que les données ont été arrondies. Par ailleurs, trois jeunes filles intérrogées ont refusé de répondre. 


\section{Mariage précoce}

Dans l'ensemble, 25,2\% des femmes âgées de 15 à 19 ans dans l'échantillon ont indiqué qu'elles étaient ou avaient été mariées. À Sikasso, ce chiffre était de $27,9 \%$, tandis que dans la région de Ségou, le taux de mariage était légèrement inférieur (23,8\%). Parmi l'ensemble de l'échantillon (adolescentes âgées de 12 à 19 ans), 15,4\% des filles interrogées à Sikasso étaient ou avaient été mariées par rapport à 14,2\% à Ségou. Une seule répondante de moins de 15 ans a déclaré être mariée. Les taux de mariage très précoce (avant l'âge de 15 ans) étaient faibles : 9,0\% des filles âgées de 15 à 19 ans à Sikasso ont indiqué qu'elles avaient été mariées avant l'âge de 15 ans par rapport à seulement 7,0\% à Ségou. La Diagramme 5 indique les proportions de femmes mariées par âge et par région et suggère que la proportion de filles mariées augmente plus rapidement après l'âge de 16 ans, et qu'à 19 ans, près de $70 \%$ et $50 \%$ des filles déclarent être ou avoir été mariées à Sikasso et Ségou, respectivement.

Dans le Tableau 4, nous examinons des caractéristiques clés du mariage précoce, telles que la proportion de filles dans des unions polygames $(30,4 \%$ à Sikasso et $23,0 \%$ à Ségou) et l'écart d'âge moyen entre les époux. Parmi les
RÉSULTAT CLÉ

$250 \%$ à 19 ans qui sont déjà mariées (échantillon complet)

filles mariées dans l'échantillon, l'écart d'âge moyen entre les époux était de 10,4 ans. Parmi les participantes, 59,3\% ont déclaré avoir un mari qui a au moins 10 ans de plus, $31,4 \%$ entre 5 et 10 ans de plus, et 9,3\% moins de 5 ans de plus ou le même âge.

De légères différences entre les régions ont été observées, avec les écarts d'âges moyens entre les époux de 11,6 ans à Sikasso et de 9,8 ans à Ségou. Le divorce et le veuvage sont rares et les proportions de filles qui sont actuellement mariées ou qui ont été mariées dans le passé étaient similaires dans les deux régions. L'étude suggère que le mariage précoce est normalisé dans ces contextes. Les filles ne sont pas conscientes des conséquences néfastes du mariage précoce (seulement $11,5 \%$ des filles ont pu identifier au moins trois conséquences néfastes liées au mariage précoce pour les filles), ni des dispositions fixant

\section{DIAGRAMME 5. Proportion de filles déjà mariées par tranches d'âge}

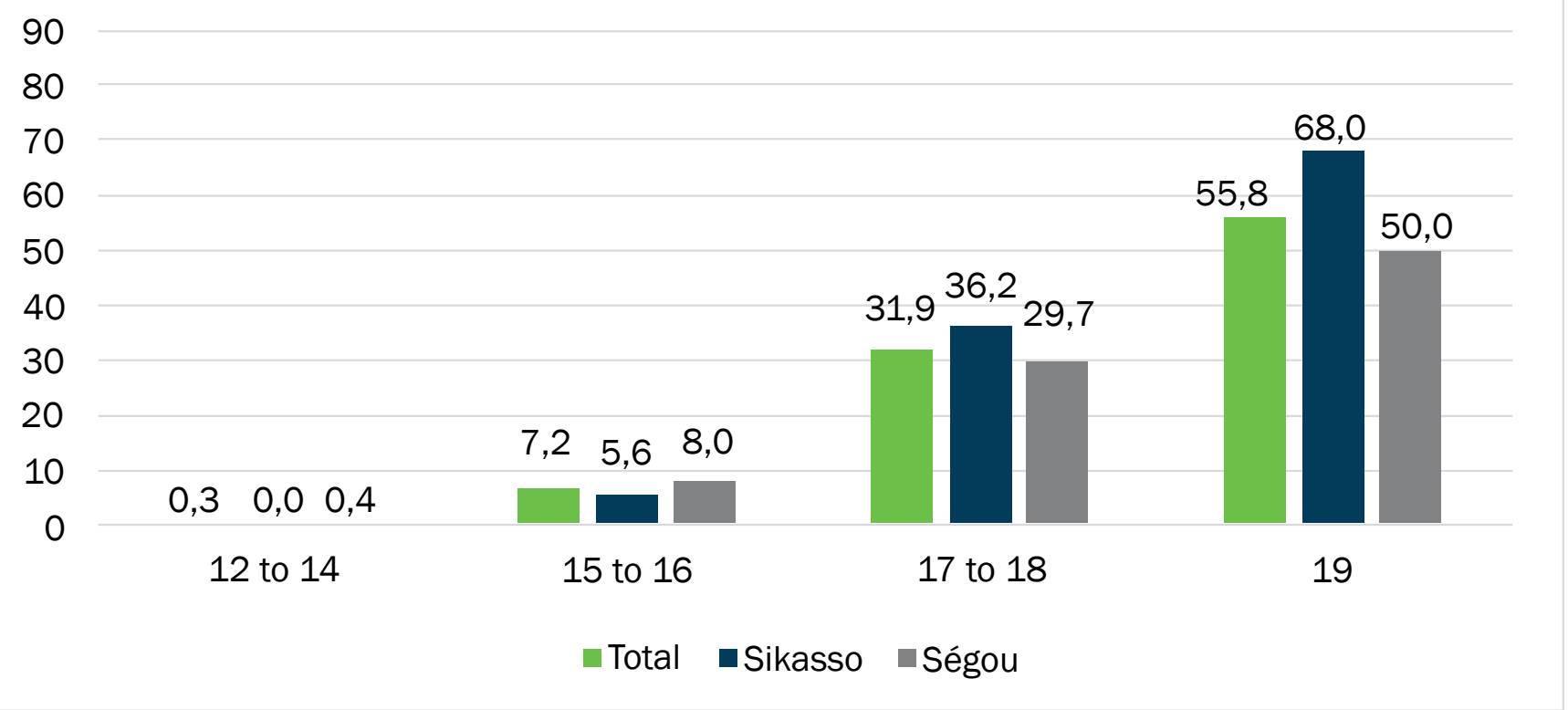


TABLEAU 4. Indicateurs clés liés au mariage parmi les filles âgées de 12 à 19 ans, par région

\begin{tabular}{|l|c|c|}
\hline & $\begin{array}{c}\text { Sikasso } \\
\mathrm{N}=300\end{array}$ & $\begin{array}{c}\text { Ségou } \\
\mathrm{N}=555\end{array}$ \\
\hline Actuellement mariées (\%) & 15,4 & 13,9 \\
\hline Actuellement mariées ou mariées auparavant (\%) & 15,4 & 23,0 \\
\hline $\begin{array}{l}\text { Dans une union polygame (parmi les filles } \\
\text { mariées) (\%) }\end{array}$ & 30,4 & 9,8 \\
\hline $\begin{array}{l}\text { Ecart d'âge moyen entre la femme et son } \\
\text { époux (parmi les filles mariées) (en années) }\end{array}$ & 11,6 & 6,3 \\
\hline $\begin{array}{l}\text { Filles sachant que l'âge légal du mariage est } \\
\text { de 16 ans pour les filles (\%) }\end{array}$ & 8,5 & 14,0 \\
\hline $\begin{array}{l}\text { Filles pouvant identifier au moins trois } \\
\text { conséquences néfastes liées au mariage pré- } \\
\text { coce pour les filles (\%) }\end{array}$ & 6,7 & 1 \\
\hline
\end{tabular}

l'âge légal du mariage (seulement 7,1\% des femmes de l'échantillon ont pu correctement identifier l'âge légal du mariage de 16 ans pour les filles au Mali). Ces chiffres pourraient être des points d'entrée importants pour initier des conversations sur le mariage précoce au niveau de la communauté. Les programmes doivent évaluer dans quelle mesure la question de mariage précoce est problématique dans le contexte local avant de lancer des initiatives visant à mettre fin à la pratique.

\section{$7,1 \%$ : Pourcentage de filles qui ont pu}

correctement identifier l'âge légal du

mariage pour les filles au Mali (16 ans)

11,5\%: Pourcentage de filles qui ont pu identi

fier au moins trois conséquences néfastes

liées au mariage précoce pour les filles

\section{0,4 ans: Ecart d'âge moyen entre les époux}

(parmi les filles mariées) 


\section{Maternité chez les adolescentes}

En Afrique de l'Ouest, il est généralement présumé que les grossesses ne se produisent que dans le cadre du mariage et que l'activité sexuelle ou les grossesses avant le mariage sont rares, voire inexistantes (seulement 4 à $6 \%$ des grossesses se produisent hors du mariage) (Center for Reproductive Law and Policy, 1999). Néanmoins, certaines études ont démontré que les rapports sexuels et les grossesses avant le mariage sont en croissance au Mali, en particulier dans les zones urbaines (Gueye, Castle et Konaté 2001). Nous avons posé aux participantes âgée de 15 ans ou plus ( $n=493$ ) quelques questions sur leurs expériences de grossesse. Nous avons découvert que 9,3\% des filles qui n'avaient jamais été mariées ( $n=365$ ) ont dit qu'elles avaient déjà été enceintes ( $n=34)$, et que $23,5 \%(n=8)$ de ces filles étaient enceintes au moment de l'entretien. L'âge moyen des filles qui n'ont jamais été mariées ayant déclaré avoir été enceintes était de 17,6 ans. Parmi les filles mariées actuellement ou dans le passé, 61,3\% d'entre elles ont signalé avoir déjà été enceintes.

La proportion élevée de filles mariées qui ont commencé à avoir des enfants peut s'expliquer en partie par un manque d'accès aux connaissances sur la santé reproductive et un manque d'accès aux services et aux contraceptifs. Parmi les filles mariées, l'utilisation de la planification familiale est limitée (17,5\% des filles mariées âgées d'au moins 15 ans ont répondu qu'elles utilisaient la planification familiale). La connaissance des méthodes de contraception est mitigée. Parmi toutes les filles interrogées âgées d'au moins 15 ans, $72,3 \%$ ont des connaissances sur le préservatif, $67,4 \%$ sur la pilule, mais $43,4 \%$ de ces filles ignoraient qu'un préservatif ne devait être utilisé qu'une seule fois, et $73,1 \%$ ignoraient à quelle fréquence prendre la pilule pour qu'elle soit efficace. Le Tableau 5 présente certains indicateurs clés liés à la santé reproductive, par région.

Parmi les filles mariées ou qui avaient été mariées, nous avons examiné l'utilisation de la planification familiale et l'autonomie des femmes dans la prise de décisions sur la
43,6\%: Pourcentage de filles qui ont des connaissances sur le VIH et qui savent que l'utilisation d'un préservatif pendant chaque rapport sexuel peut réduire le risque de contracter le VIH.

8,3\%: Pourcentage de filles ayant indiqué que leur communauté dispose d'un centre de santé adapté aux jeunes

9,3\%: Pourcentage des filles d'au moins 15 ans qui ont eu une grossesse, parmi les filles qui n'ont jamais été mariées

planification familiale. Parmi les filles mariées qui n'étaient pas enceintes au moment de l'étude $(n=89)$, seulement $16,8 \%$ utilisaient une méthode de contraception. Nous leur avons demandé si leurs maris étaient au courant qu'elles utilisaient une méthode de contraception, et $60 \%$ d'entre elles ont répondu par l'affirmative. Toutefois, il est important de noter que ces chiffres sont basés sur un très petit nombre de cas $(n=10)$. Parmi les participantes mariées et non enceintes, $21,01 \%$ ont déclaré que leurs maris ont refusé d'utiliser une méthode de planification familiale ou qu'ils ont tenté de les empêcher d'en utiliser une.

Le manque d'accès aux structures sanitaires adaptées aux jeunes peut également expliquer la faible utilisation de la planification familiale. ${ }^{5}$ Parmi les participantes âgées de plus de 15 ans, seulement 8,3\% ( $n=41)$ ont indiqué que leur communauté disposait d'un structure sanitaire adaptée aux jeunes et seulement 29,3\% ( $n=12)$ d'entre elles (soit $2,4 \%$ de toutes les participantes âgées d'au moins 15 ans) ont indiqué avoir déjà visité cette structure sanitaire dans leur communauté.

\footnotetext{
${ }^{5}$ This was asked as "Does your community have an adolescent health center/youth-friendly health facility?" If the respondent answered "yes," she was then asked "Have you ever visited the adolescent health center/youth health center"?
} 
TABLEAU 5. Indicateurs clés liés à la santé reproductive, par région

\begin{tabular}{|c|c|c|c|}
\hline & Sikasso & Segou & Total \\
\hline $\begin{array}{l}\text { Filles étant tombées enceintes, parmi les filles qui } \\
\text { étaient ou avaient été mariées (\%) }\end{array}$ & 58,7 & 62,8 & 61,3 \\
\hline $\begin{array}{l}\text { Filles étant tombées enceintes, parmi les filles } \\
\text { jamais (\%) }\end{array}$ & 9,3 & 9,3 & 9,3 \\
\hline $\begin{array}{l}\text { Recours à la planification familiale, parmi les filles } \\
\text { mariées (\%) }\end{array}$ & $(9,1)$ & $(21,9)$ & 17,5 \\
\hline Filles ayant des connaissances sur le préservatif (\%) & 67,9 & 74,5 & 72,3 \\
\hline $\begin{array}{l}\text { Filles ignorant qu'un préservatif ne doit être utilisé } \\
\text { qu'une seule fois pour être efficace (\%) }\end{array}$ & 37,5 & 46,1 & 43,4 \\
\hline Filles ayant des connaissances sur la pilule (\%) & 60,0 & 71,2 & 67,4 \\
\hline $\begin{array}{l}\text { Filles ignorant qu'il faut prendre la pilule tous les } \\
\text { jours pour qu'elle soit efficace (\%) }\end{array}$ & 68,7 & 74,6 & 73,1 \\
\hline $\begin{array}{l}\text { Filles ayant des connaissances sur le VIH et sachant } \\
\text { que l'utilisation d'un préservatif pendant chaque } \\
\text { rapport sexuel réduit le risque (\%) }\end{array}$ & 38,0 & 46,7 & 43,6 \\
\hline $\begin{array}{l}\text { Filles ayant déclaré que leur communauté dispose } \\
\text { d'un centre de santé adapté aux jeunes (\%) }\end{array}$ & 9,1 & 8,0 & 8,3 \\
\hline
\end{tabular}

Les chiffres entre parenthèses sont basés sur moins de 10 cas. 


\section{Éducation}

Un objectif important du programme MTBA est d'augmenter l'accès à la scolarisation et à la participation à une instruction formelle. Nous avons examiné le taux de scolarisation, le nombre d'années de scolarité complétées, et les compétences auto-déclarées en alphabétisation et en calcul. La Diagramme 6 montre la proportion des filles non scolarisées par âge. Les données suggèrent que le taux de scolarisation est déjà faible à l'âge de 12 ans (54,2\% des filles de 12 ans n'étaient pas scolarisées) et que les proportions des filles scolarisées chutent à partir de 15 ans et continuent à diminuer au fur et à mesure que les filles avancent en âge, pour atteindre $74,0 \%$ des filles âgées de 19 ans non scolarisées. L'examen de ces données par région révèle que la proportion de filles non scolarisées est plus élevée à Ségou qu'à Sikasso (dans l'ensemble, 64,1\% des participantes de l'échantillon à Ségou n'étaient pas scolarisées par rapport à 51,3\% à Sikasso).

Nous avons également examiné les années d'études complétées parmi les filles qui avaient été scolarisées dans les deux régions. Le nombre moyen d'années d'études achevées était de 6,2 à Sikasso et de 6,3 à Ségou. Le Tableau 6 présente les variables clés liées à l'éducation des filles dans les deux régions.

Le Tableau 7 examine les indicateurs clés liés à l'éducation des filles selon l'histoire conjugale. Les adolescentes mariées étaient moins susceptibles d'être allées à l'école que les adolescentes jamais mariées. Le mariage met fin au parcours scolaire des filles, dans la mesure où $91,2 \%$ des filles mariées ne sont pas scolarisées par rapport à 54,5\% des filles jamais mariées. Les adolescentes mariées sont moins susceptibles de savoir lire ou écrire que les filles non

\section{0,4\%: Pourcentage de filles âgées de 12 à 19 ans qui sont actuellement scolarisées.}

\section{9,9\%: Pourcentage de filles qui ont déclaré avoir} été harcelées à l'école.

6,3: Nombre moyen d'années de scolarité complétées (parmi les filles scolarisées actuellement et celles qui l'ont été dans le passé).

DIAGRAMME 6. Proportion de filles non scolarisées, par âge $(n=855)$

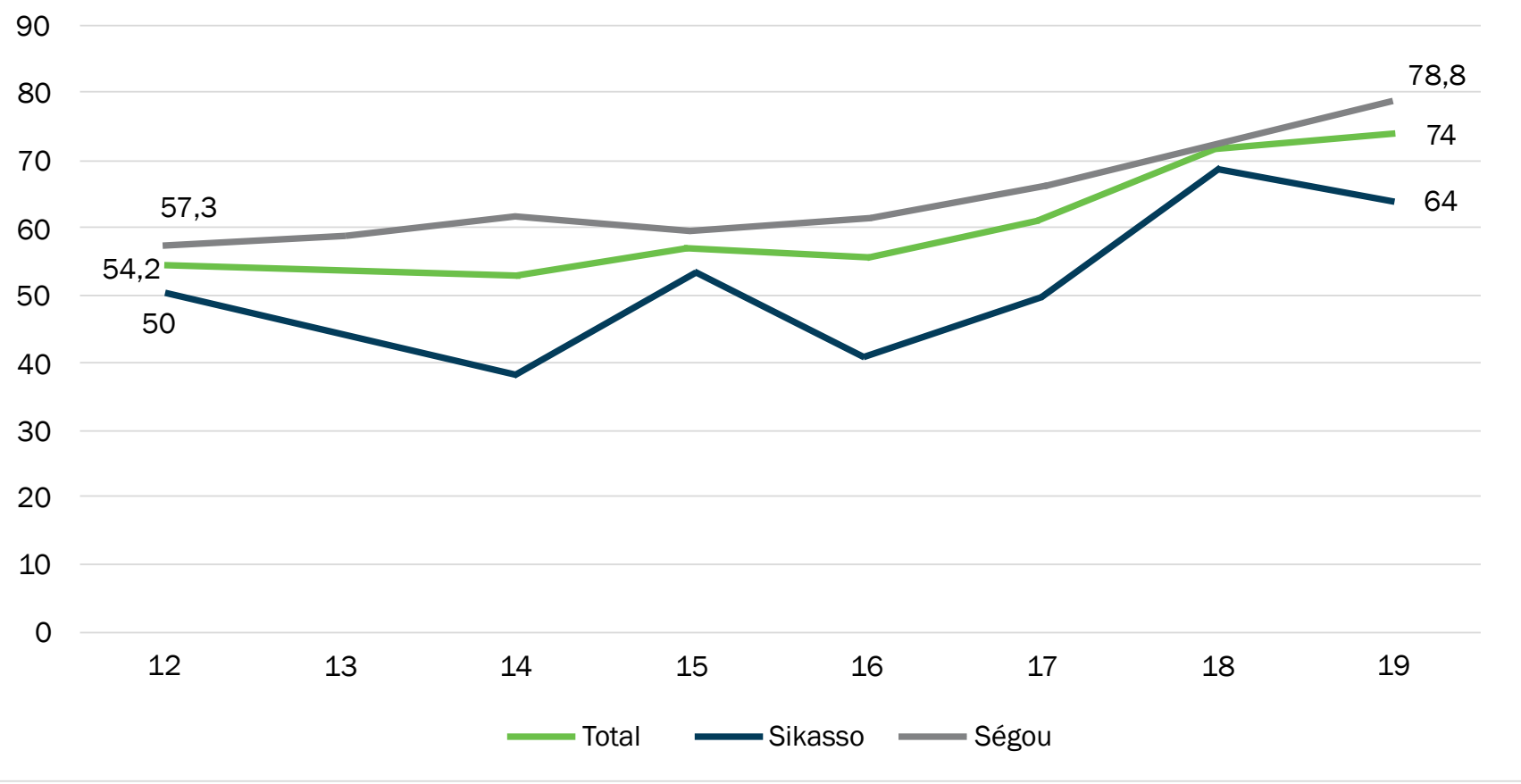


TABLEAU 6. Variables clés sur l'éducation, filles âgées de 12 à 19 ans ( $n=855)$, par région

\begin{tabular}{|l|c|c|c|}
\hline & $\begin{array}{c}\text { Sikasso } \\
\mathrm{N}=300\end{array}$ & $\begin{array}{c}\text { Segou } \\
\mathrm{N}=555\end{array}$ & $\mathrm{~N}=855$ \\
\hline Filles jamais scolarisées (\%) & 34,3 & 38,9 & 37,3 \\
\hline Filles non scolarisées actuellement (\%) & 51,3 & 54,1 & 50,6 \\
\hline $\begin{array}{l}\text { Filles qui ne savent ni lire ni écrire } \\
\text { (échantillon complet) (\%) }\end{array}$ & 45,0 & 53,0 & 7,0 \\
\hline $\begin{array}{l}\text { Filles qui ne savent ni lire ni écrire } \\
\text { (parmi celles qui sont scolarisées } \\
\text { actuellement) (\%) }\end{array}$ & 4,8 & 8,6 & \\
\hline $\begin{array}{l}\text { Filles qui ne savent ni lire ni écrire } \\
\text { (parmi celles qui sont ou ont été } \\
\text { scolarisées) (\%) }\end{array}$ & 16,7 & 25,4 & 22,2 \\
\hline
\end{tabular}

TABLEAU 7. Variables clés sur l'éducation selon le statut matrimonial, filles âgées 12 à 19 ans (n=854)

\begin{tabular}{|l|c|c|}
\hline & Ever married & Never married \\
& $\mathrm{N}=125$ & 529 \\
\hline Filles scolarisées à un moment (\%) & 50,4 & 64,7 \\
\hline Filles non scolarisées actuellement (\%) & 91,2 & 54,2 \\
\hline $\begin{array}{l}\text { Nombre d'années d'études complétées (parmi celles qui ont } \\
\text { déjà été scolarisées) (moyen) }\end{array}$ & 6,5 & 6,2 \\
\hline Filles n'étant pas capables de lire ou d'écrire (\%) & 65,6 & 47,6 \\
\hline Âge (moyen) & 17,8 & 14,8 \\
\hline
\end{tabular}

${ }^{a}$ Les différences selon la région pourraient être dues en grande partie à l'âge, car les filles mariées ont trois ans de plus de les filles jamais mariées en moyenne.

mariées (65,6\% par rapport à 47,6\%) malgré le fait qu'elles aient complété un nombre plus élevé d'années de scolarité. Parmi les filles scolarisées, 4,8\% des filles à Sikasso et 8,6\% à Ségou ont répondu qu'elles ne savaient ni lire ni écrire.

En plus de leur expérience sur la scolarité, nous avons également examiné le niveau d'alphabétisation en demandant aux participantes si elles savaient lire, écrire, faire les deux ou aucun des deux (auto-évaluation) et en leur administrant une courte évaluation de leurs compétences en lecture (Tableau 8). Le taux d'alphabétisation auto-déclaré était plus élevé à Sikasso qu'à Ségou. À Sikasso, 51,0\% des participantes ont déclaré savoir lire et écrire, par rapport à 40,6\% des filles à Ségou. Nous avons examiné ces réponses selon le niveau d'éducation des filles et avons constaté que parmi les filles scolarisées, 90,4\% et 85,8\% ont déclaré savoir lire et écrire à Sikasso et Ségou, respectivement. Parmi les filles non scolarisées actuellement, seulement 13,6\% à Sikasso et 15,1\% à Ségou ont déclaré savoir lire et écrire. En plus des données auto-déclarées, nous avons effectué une évaluation de l'alphabétisation en demandant aux

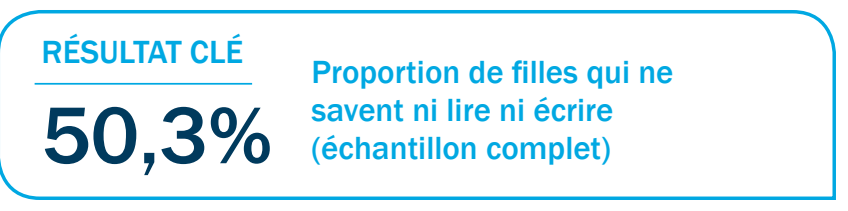

participantes de lire deux phrases en français (la langue d'enseignement). À Sikasso, 21,0\% des participantes ont réussi à lire au moins l'une des deux phrases et 19,0\% ont réussi à lire les deux. Au Ségou, ces chiffres étaient de $17,1 \%$ et $16,3 \%$, respectivement. Parmi les filles scolarisées, 35,6\% et 37,6\% pouvaient lire les deux phrases à Sikasso et à Ségou, respectivement. Ces résultats suggèrent que la qualité des écoles peut être faible. Nous avons également posé quelques questions sur les aspirations des filles en matière d'éducation afin de mieux comprendre leurs souhaits en termes d'opportunités éducatives. Parmi les filles scolarisées ( $n=536$ ), les réponses les plus fréquemment citées à la question "Quel niveau souhaitez-vous atteindre à l'école ? " étaient " au-delà du baccalauréat/de la licence " (31,0\%), "le baccalauréat/ la licence " $(18,3 \%)$, et " une école professionnelle " $(11,2 \%)$. 
TABLEAU 8. Alphabétisation auto-déclarée et évaluée, par région et statut scolaire ( $\mathrm{n=852)}$

\begin{tabular}{|c|c|c|c|c|c|c|}
\hline & \multicolumn{3}{|c|}{ Sikasso } & \multicolumn{3}{|c|}{ Ségou } \\
\hline & \multirow{2}{*}{ Total } & \multicolumn{2}{|l|}{ Statut scolaire } & \multirow{2}{*}{ Total } & \multicolumn{2}{|l|}{ Statut scolaire } \\
\hline & & Pas inscrites & Inscrites & & Pas inscrites & Inscrites \\
\hline $\begin{array}{l}\text { Filles pouvant lire et } \\
\text { écrire (auto-déclaré) }\end{array}$ & 51,0 & 13,6 & 90,4 & 40,6 & 15,1 & 85,8 \\
\hline $\begin{array}{l}\text { Filles pouvant lire l'une } \\
\text { des deux phrases en } \\
\text { français }\end{array}$ & 21,0 & 7,1 & 35,6 & 17,1 & 7,4 & 34,5 \\
\hline $\begin{array}{l}\text { Filles pouvant lire les } \\
\text { deux phrases en français }\end{array}$ & 19,0 & 3,2 & 35,6 & 16,3 & 4,6 & 37,6 \\
\hline
\end{tabular}

Nous avons également demandé aux filles le niveau de scolarité que leurs parents voudraient les voir atteindre. La réponse la plus fréquente était " jusqu'au niveau que je veux " $(24,4 \%)$, " au-delà du baccalauréat/ de la licence " $(23,1 \%)$ et " le baccalauréat/ la licence " $(14,9 \%)$. Un nombre important de filles ( $n=97 ; 18,1$ ont répondu qu'elles ignoraient le niveau de scolarité que leurs parents voudraient les voir atteindre. 


\section{Moyens de subsistance}

Les études suggèrent que la pauvreté et le manque d'opportunités pour la production de revenus sont des facteurs clés qui influencent le mariage précoce dans certains contextes (Malhotra et al, 2011). Nous avons interrogé les participantes sur leurs expériences de travail et sur le genre de travail qu'elles ont accompli. Les données suggèrent que de nombreuses filles participent d'ores et déjà à des activités génératrices de revenus : $48,6 \%$ des participantes âgées de 12 à 19 ans ont déclaré avoir travaillé pour gagner de l'argent. Parmi les participantes plus âgées (entre 15 et 19 ans), cette proportion était de 57,0\%. Parmi celles qui avaient déjà travaillé $(n=413)$, nous avons demandé si elles participaient actuellement à des activités génératrices de revenus, 42,6\% d'entre elles ont répondu par l'affirmative. La proportion des filles participant actuellement à des activités génératrices de revenus était plus élevée à Sikasso (51,5\%) qu'à Ségou $(38,4 \%)$.

Nous avons interrogé les filles qui travaillaient sur le l'activité effectuée, les horaires et le revenu mensuel. La profession la plus fréquemment citée était commerçante $(48,3 \%)$, suivie de travailleuse agricole $(26,1 \%)$, et femme de ménage (15,9\%). Quelques différences régionales ont été observées : à Sikasso, travailleuse agricole était la profession la plus citée $(47,0 \%)$, suivie par commerçante $(41,2 \%)$, tandis qu'à Ségou, les professions les plus citées commerçante $(52,8 \%)$ et femme de ménage $(23,1 \%)$. Parmi toutes les filles qui travaillent pour gagner de l'argent, le revenu mensuel moyen était 15659 FCFA (soit environ 26 dollars américains).

Dans l'ensemble, 25,2\% des filles de l'échantillon de référence ont déclaré faire des économies pour l'avenir. Presque aucune des participantes à l'étude $(0,5 \%)$ n'a déclaré avoir un compte d'épargne dans une banque. La majorité des participantes ont indiqué qu'elles conservaient leurs économies chez elles $(70,2 \%)$ ou chez un ami ou un membre de la famille (28,8\%). Les raisons les plus fréquemment évoquées pour justifier les économies étaient l'achat

\section{2,6\%: Le pourcentage de filles qui participent actuellement à des activités génératrices de revenus (parmi les filles de 12 à 19 ans qui ont déclaré avoir travaillé). \\ 25,2\%: Le pourcentage de filles qui ont déclaré avoir épargné pour l'avenir}

de vêtements, de chaussures, ou d'autres articles personnels $(46,0 \%)$, le mariage $(26,5 \%)$, ou une urgence $(18,1 \%)$.

Les données sur les stratégies de subsistance adoptées par les adolescentes à Sikasso et à Ségou montrent que les filles commencent à participer à des activités génératrices de revenus très jeunes et que près de la moitié des filles travaillent pour gagner de l'argent. Les revenus déclarés par les filles comparés au seuil de pauvreté ${ }^{6}$ (une estimation du montant de revenu nécessaire pour maintenir un niveau de vie élémentaire minimum), suggèrent que les filles qui travaillent peuvent gagner suffisamment d'argent pour subvenir à leurs besoins personnels. Les taux d'épargne déclarés sont également élevés avec des différences importantes entre les zones d'intervention et les zones de comparaison.

Malgré ces fortes tendances à faire des économies chez les filles, les données suggèrent que l'accès à des institutions d'épargne sûres est limité et que les jeunes ont recours à des moyens informels pour protéger leurs revenus, en comptant sur les membres de leurs familles.

\footnotetext{
${ }^{6}$ Selon la Banque Mondiale, en 2010, 49,3\% de la population mondiale vivaient avec moins de \$1,90 par jour http://povertydata.worldbank.org/poverty/country/MLI
} 
TABLEAU 9. Indicateurs clés sur les moyens de subsistance, par région

\begin{tabular}{|c|c|c|c|}
\hline & Sikasso & Ségou & Total \\
\hline $\begin{array}{l}\text { Filles âgées de } 12 \text { à } 19 \text { ans ayant } \\
\text { travaillé pour gagner de l'argent (\%) }\end{array}$ & 44,1 & 51,0 & 51,4 \\
\hline $\begin{array}{l}\text { Filles âgées de } 15 \text { à } 19 \text { ans) ayant } \\
\text { travaillé pour gagner de l'argent (\%) }\end{array}$ & 54,2 & 58,5 & 57,0 \\
\hline $\begin{array}{l}\text { Filles travaillant au moment de l'étude } \\
\text { pour gagner de l'argent (parmi celles } \\
\text { qui ont déjà travaillé) (\%) }\end{array}$ & 51,5 & 38,4 & 42,6 \\
\hline $\begin{array}{l}\text { Filles travaillant en tant que } \\
\text { commerçantes (parmi celles qui } \\
\text { travaillent actuellement) (\%) }\end{array}$ & 41,2 & 52,8 & 48,3 \\
\hline $\begin{array}{l}\text { Filles travaillant dans l'agriculture } \\
\text { (parmi celles qui travaillent } \\
\text { actuellement) }(\%)\end{array}$ & 47,0 & 13,0 & 26,1 \\
\hline $\begin{array}{l}\text { Filles gagnant de l'argent en faisant } \\
\text { du travail domestique (parmi celles qui } \\
\text { travaillent actuellement) (\%) }\end{array}$ & 4,4 & 23,1 & 15,9 \\
\hline $\begin{array}{l}\text { Revenu moyen par mois (parmi celles } \\
\text { qui travaillent actuellement) (FCFA } \\
\text { (USD)) }\end{array}$ & $\begin{array}{c}13,113 \\
(\$ 22,50)\end{array}$ & $\begin{array}{l}17,223 \\
(\$ 29,62)\end{array}$ & $\begin{array}{l}15,659 \\
(\$ 26,88)\end{array}$ \\
\hline $\begin{array}{l}\text { Filles déclarant épargner pour l'avenir } \\
(\%)\end{array}$ & 23,7 & 26,0 & 25,2 \\
\hline
\end{tabular}




\section{La vie sociale des adolescentes}

Les interventions visant à retarder l'âge du mariage doivent s'efforcer de comprendre le contexte social dans lequel vivent les filles, notamment les normes de genre et les attitudes dominantes à l'égard des femmes. Pour mieux comprendre le contexte social dans ces communautés, nous nous sommes penchés sur les attitudes équitables liées au genre, en posant aux participantes des questions sur les droits des filles, la place des filles dans leurs familles par rapport aux garçons, les attitudes envers la violence contre les femmes, et les notions de masculinité. Nous avons examiné ces indicateurs selon le statut matrimonial des participantes, en partant de l'hypothèse que les filles mariées pourraient avoir des perspectives différentes de celles des filles n'ayant jamais été mariées. Nous avons également examiné les données par région. Le Tableau 10 présente les résultats. Dans toutes les catégories présentées, les réponses illustrent uniformément que les participantes avaient des avis très inégaux sur les rôles appropriés des femmes. La majorité écrasante des participantes jugeaient que le rôle des femmes était de s'occuper des tâches ménagères, de faire la cuisine et qu'elles devaient prendre soin de leur maison. Les participantes jugeaient également qu'une femme devait obéir à son mari et accepter la violence afin de préserver l'unité de la cellule familiale, même si moins de participantes jugeaient que parfois, il était légitime de battre une femme.

En plus des attitudes liées au genre, nous avons également cherché à comprendre la vie sociale des filles dans ces communautés. Nous demandé aux filles quelle était leur expérience en matière de moqueries ou de harcèlement, puis avons comparé les réponses dans les régions de Sikasso et Ségou. La Diagramme 8 présente les réponses aux indicateurs clés. Dans ces communautés, plus d'un tiers des participantes ont déclaré avoir été victimes de moqueries ou de harcèlement. Parmi elles, le harcèlement se produisait le plus fréquemment à l'extérieur de leur domicile (73,7\% à Sikasso et 72,7\% à Ségou) et moins fréquemment à l'école (24,6\% à Sikasso et $27,7 \%$ à Ségou).

Des recherches antérieures nous ont appris que lorsque les filles grandissent, leur monde se rétrécit par rapport à celui des hommes (Hallman et al. 2015), et nos résultats
40,1\%: Pourcentage des filles qui ont répondu qu'elle ne sont pas membres d'une association ou d'un club.

98,8\%: Pourcentage des filles qui sont "d'accord" ou "tout à fait d'accord" avec l'assertion qu'une femme devrait toujours obéir à son mari.

93,9\%: Pourcentage de filles qui sont "d'accord" ou "tout à fait d'accord" avec l'assertion qu'une femme devrait supporter la violence afin de garder sa famille unie.

indiquent que les filles incluses dans notre étude ont une mobilité limitée. Au total, $66,6 \%$ des participantes ont déclaré qu'elles n'avaient pas le droit de sortir de la maison après le coucher du soleil (pas de différence entre les deux régions). Nous avons constaté qu'un nombre plus faible de filles âgées de 15 ans ou plus ont déclaré qu'elles étaient autorisées à seules après le coucher du soleil $(26,8 \%)$ par rapport aux filles âgées de moins de 15 ans (33,9\%). La majorité des participantes (91,2\% à Sikasso et 90,3\% à Ségou) ont déclaré que leurs parents ou leurs tuteurs s'inquiétaient pour leur sécurité.

Les résultats suggèrent que dans ces communautés, il existe des normes de genre traditionnelles entourant le mariage et les rôles des femmes au sein de la famille. Les participantes ont souligné que ni les garçons ni les filles n'avaient le droit de refuser un mariage arrangé. Dans les deux régions et parmi les filles mariées et non mariées, les participantes étaient d'accord pour dire que le rôle le plus important pour une femme était de s'occuper de sa maison, que les femmes devaient supporter la violence afin de préserver l'unité de la cellule familiale, et que les femmes devaient toujours obéir à leurs maris. Les filles qui n'ont jamais été mariées étaient moins susceptibles que les filles mariées d'affirmer que les femmes étaient seules responsables du contrôle des naissances, même si la moitié le pensait. Les interventions visant à retarder l'âge du mariage dans ces communautés doivent s'efforcer d'aborder les normes de genre prédominantes qui limitent l'autonomie et les opportunités pour les adolescentes. 
TABLEAU 10. Attitudes sur l'égalité entre les sexes, filles âgées de 12 à 19 ans

\begin{tabular}{|c|c|c|c|c|}
\hline & \multicolumn{4}{|c|}{$\begin{array}{l}\text { Pourcentage de filles qui étaient } \\
\text { " d'accord " ou " tout à fait d'accord " }\end{array}$} \\
\hline & \multicolumn{2}{|c|}{ Sikasso } & \multicolumn{2}{|c|}{ Ségou } \\
\hline & $\begin{array}{l}\text { Actuellement } \\
\text { mariées ou } \\
\text { mariées dans le } \\
\text { passé }\end{array}$ & Jamais mariées & $\begin{array}{l}\text { Actuellement } \\
\text { mariées ou } \\
\text { mariées dans le } \\
\text { passé }\end{array}$ & Jamais mariées \\
\hline & $N=46$ & $N=253$ & $N=79$ & $N=476$ \\
\hline \multicolumn{5}{|l|}{ Décision sur le mariage } \\
\hline $\begin{array}{l}\text { Les garçons n'ont pas le droit de refuser un } \\
\text { mariage arrangé }\end{array}$ & 93,5 & 94,1 & 987 & 96.2 \\
\hline $\begin{array}{l}\text { Les filles n'ont pas le droit de refuser un mariage } \\
\text { arrangé }\end{array}$ & 93,5 & 94,8 & 98,7 & 97,4 \\
\hline \multicolumn{5}{|l|}{ Rôles liés au genre } \\
\hline $\begin{array}{l}\text { Le rôle le plus important d'une femme est de } \\
\text { s'occuper du ménage et de faire la cuisine pour sa } \\
\text { famille }\end{array}$ & 93,5 & 94,1 & 91,1 & 91,4 \\
\hline $\begin{array}{l}\text { La femme doit assumer la responsabilité d'éviter de } \\
\text { tomber enceinte }\end{array}$ & 69,6 & 55,3 & 77,2 & $58,1 * *$ \\
\hline $\begin{array}{l}\text { Si quelqu'un insulte un homme, il doit défendre sa } \\
\text { réputation, par la force si nécessaire }\end{array}$ & 65,2 & 72,1 & 79,7 & 72,8 \\
\hline Une femme doit toujours obéir à son mari. & 100,0 & 98,4 & 100,0 & 98,7 \\
\hline $\begin{array}{l}\text { Une femme doit avoir le droit de divorcer de son } \\
\text { mari }\end{array}$ & 26,1 & 32,1 & 38,5 & 35,0 \\
\hline \multicolumn{5}{|l|}{ Acceptation de la violence } \\
\hline $\begin{array}{l}\text { Il y a des moments où une femme mérite d'être } \\
\text { battue }\end{array}$ & 26,1 & 36,0 & 36,7 & 37,5 \\
\hline $\begin{array}{l}\text { Une femme doit supporter la violence afin de pré- } \\
\text { server l'unité de la cellule familiale }\end{array}$ & 95,6 & 89,6 & 97,5 & 95,4 \\
\hline $\begin{array}{l}\text { Une femme ne peut pas refuser d'avoir des rap- } \\
\text { ports sexuels avec son mari. (Question posée aux } \\
\text { participantes âgées d'au moins } 15 \text { ans) }\end{array}$ & 67,4 & 24,3 & 62,0 & 30,1 \\
\hline $\begin{array}{l}\text { Quand une femme est violée, elle est généralement } \\
\text { en faute pour s'être mise dans cette situation. } \\
\text { (Question posée aux répondantes âgées d'au moins } \\
15 \text { ans) }\end{array}$ & 34,8 & 15,5 & 45,6 & 18,1 \\
\hline
\end{tabular}

DIAGRAMME 8. Expérience de moqueries ou de harcèlement

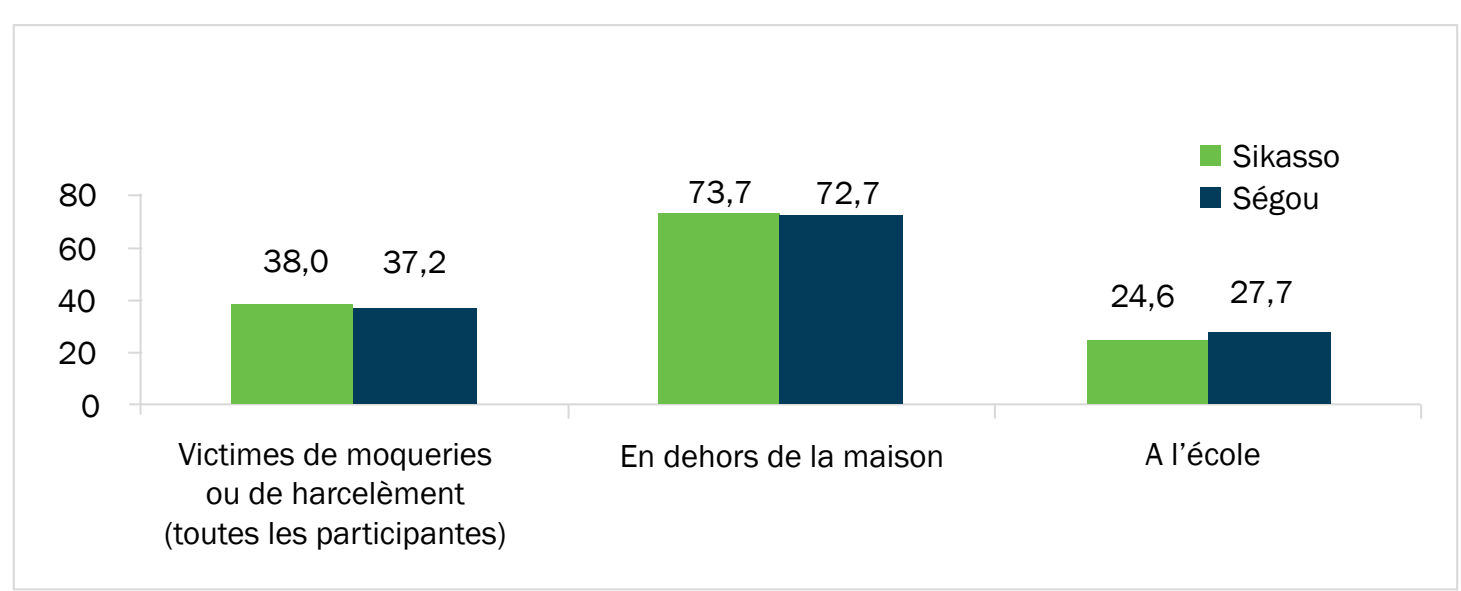




\section{Discussion}

Afin d'identifier les domaines où les interventions pourraient améliorer les conditions des filles au Mali, nous nous sommes penchés sur la vie de ces adolescentes, y compris leurs connaissances et leurs expériences en matière de santé reproductive, d'éducation, de résultats scolaires, et de leurs expériences et leurs attitudes à l'égard du mariage. L'étude des filles dans des communautés spécifiques à Sikasso et à Ségou, nous a permis d'avoir une compréhension plus nuancée de leur vie dans ces communautés que ce que montrent les études disponibles, comme l'EDS, ainsi qu'une mesure de référence pour évaluer l'impact du programme.

Un certain nombre de résultats, mis en exergue ci-dessous, sont intéressants et se prêtent à un examen approfondi au fur et à mesure de la mise en œuvre du programme MTBA :

- Nos résultats montrent que même si les filles dans notre échantillon sont plus susceptibles d'être plus instruites que leurs parents, une proportion importante n'a jamais été scolarisée (37,3\%). Parmi celles qui sont allées à l'école, une proportion importante (16,7\% à Sikasso et $25,4 \%$ à Ségou) ne sait ni lire ni écrire, suggérant que la qualité des écoles peut être faible. Les interventions qui ne ciblent que les filles scolarisées et/ ou les interventions reposant sur la capacité des filles à lire et à écrire passeront à côté des certaines sous-populations importantes qui peuvent être particulièrement vulnérables au mariage précoce.

- Les écarts d'âge entre les époux sont relativement importants parmi les filles, sachant que plus de la moitié des filles mariées ont indiqué avoir un partenaire âgé d'au moins 10 ans de plus qu'elles. Des études suggèrent que les filles dont les partenaires sont beaucoup plus âgés qu'elles sont moins susceptibles de prendre des décisions, y compris des décisions liées à la fécondité, ce qui peut avoir des implications pour les interventions qui abordent l'accès et l'utilisation de services en santé reproductive. En outre, des écarts d'âge importants impliquent la forte probabilité que les époux décèderont avant leurs épouses, ce qui signifie que les filles plus jeunes que leurs maris deviendront probablement veuves ou mères célibataires plus tard dans la vie.
- Concernant la fécondité et la procréation, nos résultats montrent qu'une proportion importante de filles n'ayant jamais été mariées ont déclaré avoir été enceintes $(9,3 \%)$ et que parmi les filles mariées, la grossesse suit rapidement. Parmi les filles mariées, l'utilisation de la planification familiale est limitée $(82,5 \%$ des filles mariées âgées d'au moins 15 ans ont répondu qu'elles n'utilisaient pas la planification familiale et $61,3 \%$ ont déclaré avoir été enceintes). Ces résultats suggèrent que les interventions pourraient chercher des stratégies pour améliorer l'accès et l'utilisation de la contraception dans ces communautés et sensibiliser les maris sur la planification familiale.

- Nous avons souhaité savoir si dans notre échantillon, une proportion importante de filles participait à des activités génératrices de revenus ( $57,0 \%$ des filles âgées de 15 à 19 ans ont déclaré avoir travaillé pour gagner de l'argent), le commerce étant une activité génératrice de revenus répandue. L'activité économique de ce groupe suggère qu'il est possible de mettre à contribution les compétences et les motivations des filles pour qu'elles puissent participer à l'économie. II est également nécessaire d'inclure un volet sur la formation financière dans les programmes afin que les filles apprennent à gérer leurs revenus. Ces résultats peuvent également contribuer à expliquer les taux de mariage plus faibles que prévus chez les filles de notre échantillon par rapport aux données publiées par I'EDS : les filles qui ont la possibilité de participer à des activités génératrices de revenus comme alternative au mariage pourraient avoir plus d'autonomie en décidant de retarder leur mariage.

Les données de l'étude de référence indiquent que même si la prévalence du mariage précoce chez les adolescentes est plus faible dans notre échantillon à que ce qu'indiquent les enquêtes représentatives nationales, d'autres corrélats du mariage précoce sont identiques et fréquents. Les différences observées peuvent être attribuées aux différences dans le pays ou à des tendances temporelles. Comme le montre le rapport initial, le mariage précoce est en baisse au Mali. Cette tendance est peut être représentative d'un changement de trajectoire générale en matière de développement. 
II convient de tenir compte de certaines limites. Premièrement, la conception de l'étude n'a pas permis la répartition aléatoire des communautés ; il est donc possible que les communautés désignées pour faire partie du programme MTBA vivent dans de meilleures conditions que les communautés typiques des régions de Ségou et de Sikasso en raison des critères utilisés dans la sélection des sites du programme. Par exemple, il est possible que les filles dans ces communautés aient un niveau d'éducation plus élevé parce que la présence d'écoles secondaires dans ces communautés était un des critères de sélection. Une autre limite porte sur le calendrier de collecte des données. Le travail de terrain a été effectué pendant la saison des pluies au Mali, ce qui a pu influencer la sélection de filles qui étaient disponibles. Pendant la saison des pluies, certaines migrantes retournent dans leurs communautés pour participer aux activités agricoles, ou encore certaines filles ne peuvent pas retourner chez elles à cause de la pluie et des mauvaises conditions routières. Une autre limite potentielle est la subjectivité de l'auto-déclaration. Notre étude pose un certain nombre de questions sensibles, y compris des questions sur le mariage précoce, qui est illégal pour les filles âgées de moins de 16 ans au Mali. Les filles ont peutêtre été réticentes à répondre aux questions sur les sujets sensibles tels que le mariage précoce et l'activité sexuelle.

Malgré ces limites, cette étude de référence fournit des informations riches sur les expériences vécues par les filles dans ces communautés et met en évidence un certain nombre de résultats intéressants que les programmes peuvent prendre en compte pendant la conception et la mise en œuvre des interventions visant à améliorer la vie des adolescentes à Ségou et à Sikasso. La collecte de données supplémentaires, y compris des données qualitatives et des études à mi-parcours et en fin de parcours, apporteront davantage d'informations et nous donneront une meilleure idée de l'impact du programme MTBA sur les filles dans ces communautés. 


\section{Annexe 1}

L'intervention du programme MTBA au Mali est mise en œuvre par Oxfam Novib et Save the Children dans deux régions (Sikasso et Ségou) et compte 2 districts à Sikasso (Sikasso et Yorosso) et 3 districts à Ségou (Ségou, San et Bla). Nous avons utilisé une comparaison appariée à deux niveaux au Mali basée sur des études transversales répétées. Nous avons sélectionné des zones d'intervention et des zones de comparaison (chacune de ces zones représentant $50 \%$ de l'échantillon total pour l'étude de référence basée sur les villages (ZR). Sur la base d'un échantillon cible de 800 filles (40 groupes de 20 filles) nous avons estimé un effet détectable minimum de $15 \%$ pour la proportion des filles âgées de 12 à 19 ans mariées à la fin de l'étude.
Les données de l'EDS de 2012/2013 indiquent des estimations de la valeur plausible minimale et maximale de l'âge des filles au premier mariage au Mali. Les données de l'EDS révèlent que la proportion des filles âgées de 15 à 19 ans qui sont actuellement mariées ou qui sont en union varie de $39 \%$ à $56 \%$. Bien que ces chiffres varient, nous utiliserons ces valeurs et estimerons qu'un total de 20 filles par village (ZR) seront interrogées. Nous estimons qu'avec $\mathrm{n}=20$ filles par village/ZR et 40 grappes au total, la puissance statistique devrait s'élever à $80 \%$ ou plus.

DIAGRAMME 1. Calcul de la puissance pour la taille de l'échantillon au Mali

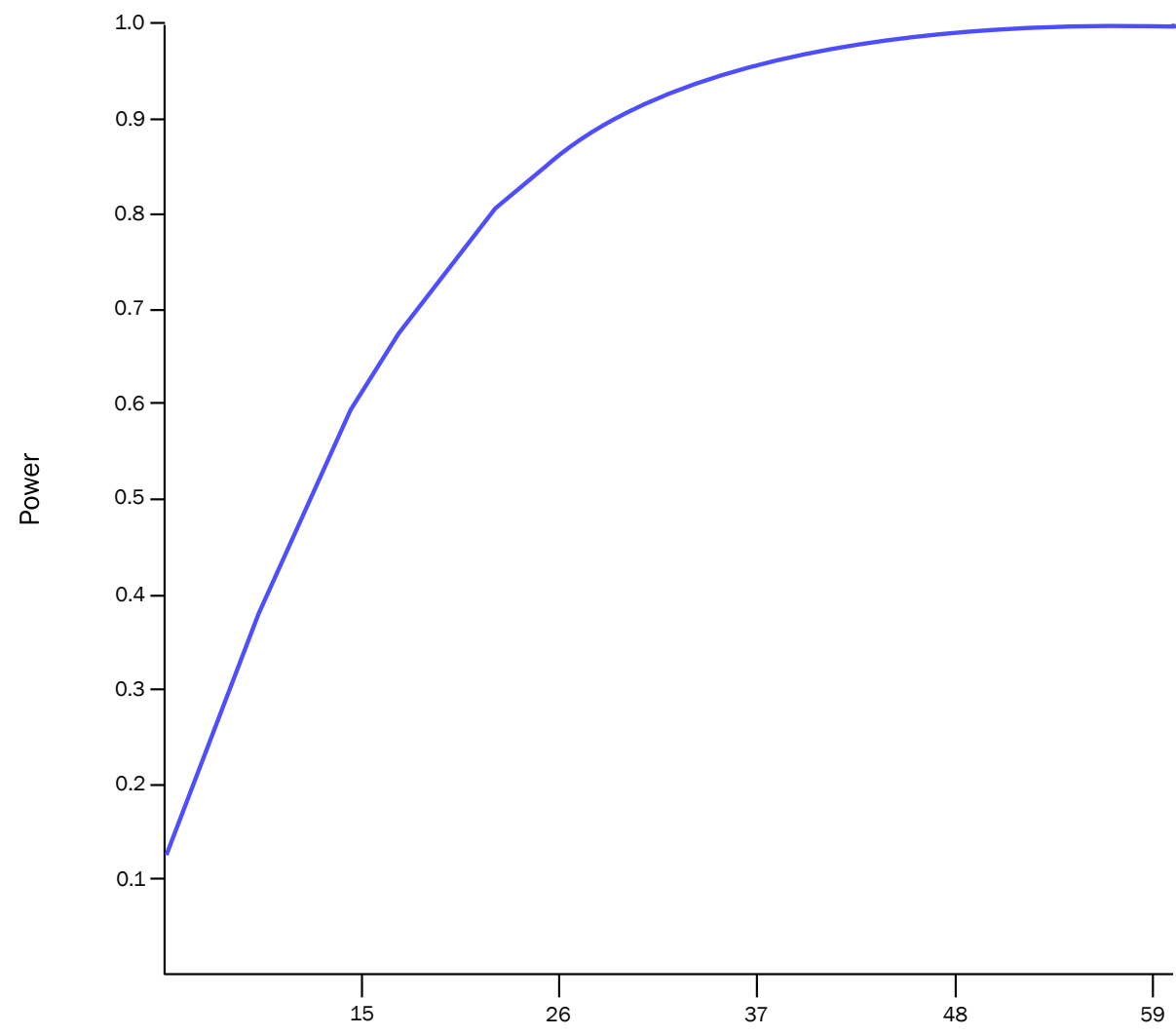

$\alpha=0.050$

${ }^{\phi} \mathrm{E}=0.550000$

${ }^{\phi} \mathrm{C}=0.400000$

lower plausible value $=0.390000$

upper plausible value $=0.560000$

$n=20$

Total number of clusters

Ce calcul présume que le groupe est le village (ZR); la valeur plausible maximale et la valeur plausible minimale ont été calculées en utilisant la proportion des filles âgées de 15 à 19 ans qui sont actuellement mariées (données de l'EDS, 2012/13) 


\section{Annexe 2}

TABLEAU 1. Indicateurs clés, filles âgées de 12 à 19 ans dans les zones d'intervention et de comparaison

\begin{tabular}{|l|c|c|}
\hline & Intervention & Comparaison \\
\hline & $\mathrm{N}=426$ & $\mathrm{~N}=429$ \\
\hline Actuellement mariées (\%) & 12,2 & 16,6 \\
\hline Actuellement mariées ou mariées dans le passé (\%) & 12,4 & 24,6 \\
\hline Dans une union polygame (parmi les filles mariées) (\%) & 27,4 & \multirow{2}{*}{$9,5^{\star}$} \\
\hline $\begin{array}{l}\text { Ecart d'âge moyen entre les époux (parmi les filles } \\
\text { mariées) (ans) }\end{array}$ & 11,5 & 42,2 \\
\hline Filles jamais scolarisées (\%) & 32,4 & 63,2 \\
\hline Filles non scolarisées actuellement (\%) & 55,9 & 56,1 \\
\hline Filles ne pouvant pas lire ou écrire (échantillon complet) (\%) & 44,1 & 7,6 \\
\hline $\begin{array}{l}\text { Filles ne pouvant pas lire ou écrire (parmi les filles } \\
\text { scolarisées actuellement (\%) }\end{array}$ & 6,4 & 42,2 \\
\hline Filles jamais scolarisées (\%) & 32,4 & \\
\hline
\end{tabular}

$* * * p<.001, * * p<.01, * p<.05$. 


\section{Références}

Cellule de planification et de statistique (CPS/SSDSPF), Institut national de la statistique (INSTAT/MPATP), INFO-STAT et ICF International, 2014. Enquête démographique et de santé au Mali 2012-2013. Rockville, Maryland, USA : CPS, INSTAT, INFO-STAT et ICF International.

Center for Reproductive Law and Policy. 1999. Reproductive Rights of Young Girls and Adolescents in Mali: A Shadow Report. New York: Center for Reproductive Law and Policy.

Gueye M, Castle S, Konaté MK. 2001. Moment des premiers rapports sexuels des adolescents du Mali et implications pour la pratique contraceptive. Perspectives Internationales sur le Planning Familial, 2001: 2-9.

Hallman, K., N. Kenworthy, J.A. Diers, N. Swan, and B. Devnarain. 2015. The shrinking world of girls at puberty: Violence and genderdivergent access to the public sphere among adolescents in South Africa. Global Public Health 10(3): 279-295.

Malhotra, A., A. Warner, A. McGonagle, and S. Lee-Rife. 2011. Solutions to end child marriage: What the evidence shows. The International Center for Research on Women, Washington, DC.

Mensch, B.S., J. Bruce, and M.E. Greene. 1998. The Uncharted Passage: Girls' Adolescence in the Developing World. New York: Population Council.

UNESCO Institute for Statistics, Global Databases, 2014. http://stats.uis.unesco.org/unesco/tableviewer/document. aspx?Reportld=143.

United Nations Children's Fund (UNICEF). 2011. State of the World's Children 2011: Adolescence, An age of Opportunity. New York: UNICEF. 